\title{
THE
}

10-1-1985

\section{Spin Chains in a Field: Crossover from Quantum to Classical Behavior}

\author{
J. B. Parkinson \\ Jill C. Bonner \\ University of Rhode Island
}

Follow this and additional works at: https://digitalcommons.uri.edu/phys_facpubs

Terms of Use

All rights reserved under copyright.

\section{Citation/Publisher Attribution}

John B. Parkinson and Jill C. Bonner. Spin chains in a field: Crossover from quantum to classical behavior. Phys. Rev. B 32, 4703 (1985).

Available at: http://dx.doi.org/10.1103/PhysRevB.32.4703

This Article is brought to you for free and open access by the Physics at DigitalCommons@URI. It has been accepted for inclusion in Physics Faculty Publications by an authorized administrator of DigitalCommons@URI. For more information, please contact digitalcommons-group@uri.edu. 


\title{
Spin chains in a field: Crossover from quantum to classical behavior
}

\author{
J. B. Parkinson* and J. C. Bonner ${ }^{\dagger}$ \\ Department of Physics and Astronomy, Michigan State University, East Lansing, Michigan 48824
}

(Received 11 February 1985)

\begin{abstract}
Extensive numerical studies have been performed on Heisenberg antiferromagnetic chains of spin $\frac{1}{2}$ (up to $\left.N=20\right)$, spin $1(N=14)$, spin $\frac{3}{2}(N=10)$, and spin $2(N=8)$. With use of the Lanczös technique, primarily, the two lowest-lying eigenvalues have been calculated for all values of wave vector $q$ and all values of magnetization $\left(S_{T}^{z}\right)$ up to saturation for each chain. From a knowledge of the eigenvector corresponding to the lowest eigenstate for each $S_{T}^{z}$, the $T=0$ spin-pair correlation functions have also been calculated as a function of field. We find a most unusual quantumclassical crossover phenomenon. It shows up in greatest detail in the field-dependent dispersion spectra, but the consequences are consistently manifested in the behavior of the $T=0$ magnetization isotherms and in the correlations both in real space and the Fourier transforms in $q$ space. The additional data relevant to behavior in a field have allowed us to extend previous numerical studies of Heisenberg antiferromagnetic chains with higher spin whose purpose was to examine the validity of the Haldane conjecture. The Haldane conjecture implies that Heisenberg antiferromagnetic chains with integer spin have a gap in their excitation spectrum whereas chains with half-integer spin do not. While no feature of our extended investigations is in conflict with the conjecture, unusual features associated apparently with very slow convergence make the outcome less than conclusive. It appears that calculations on significantly longer chains are required to observe with confidence the large- $N$ asymptotic limiting behavior.
\end{abstract}

\section{INTRODUCTION}

The study of quantum-spin chains continues to be of interest $^{1}$ and to bring forth surprises. For example, the static properties of $X X Z$ spin chains for various values of spin $S$ were believed to be well understood as a result, principally, of exact analytic (Bethe-ansatz) calculations for spin $\frac{1}{2}$ and finite-chain extrapolations for $\frac{1}{2} \leq S \leq \frac{5}{2}$. Recently, however, Haldane ${ }^{2}$ has conjectured the existence of a radically different type of $(T=0)$ phase behavior for the class of integer-spin $X X Z$ chains in comparison with the class of half-integer-spin $X X Z$ chains.

Consider the Hamiltonian

$$
H=J \sum_{i=1}^{N}\left(S_{i}^{x} S_{i+1}^{x}+S_{i}^{y} S_{i+1}^{y}+\lambda S_{i}^{z} S_{i+1}^{z}\right),
$$

where the sign in front of the exchange constant $J$ denotes antiferromagnetism, and $\lambda$ is the $X X Z$ anisotropy parameter $(\lambda=0$ corresponds to the $X Y$ model, $\lambda=1$ to the Heisenberg antiferromagnet, and $\lambda>1$ to uniaxial, Isinglike anisotropy). A sketch of the lowest-lying energy levels according to predictions of Haldane is shown as Fig. 1. The class of half-integer-spin chains is expected to show the same $T=0$ phase behavior (see Fig. 1) as the familiar exactly solvable ${ }^{3} S=\frac{1}{2}$ case. For $0 \leq \lambda \leq 1$, the spectrum is gapless with algebraic decay of order in the ground state characterized by continuously varying critical exponents $\eta^{z}$ and $\eta^{x}=\eta^{\perp}$. This Baxter line terminates in an essential singularity at $\lambda=1$, and for $\lambda>1$ a gap opens up to an excited-state continuum. The ground state is now doubly degenerate with long-range order (LRO). For the class of integer-spin chains, however, the gapless
(Kosterlitz-Thouless) phase for $\lambda<1$ and the $T=0$ ordered "antiferromagnetic" phase are still present, but a new phase appears for $\lambda_{1}<\lambda<\lambda_{2}$ which encompasses the Heisenberg point $\lambda=1$. This novel phase has a nonordered singlet ground state (exponential decay of the correlation functions) and an energy gap to the excited-state continuum.

The Haldane picture is in conflict with Kubo-Anderson spin-wave theory, is surprising in the light of smoothness-universality considerations, and suggests that the approach to the classical (spin- $\infty$ ) limit from the quantum limit of low spin value may show unexpected complications. Earlier finite-chain studies involved diagonalization of the $(2 S+1)^{N} \times(2 S+1)^{N}$ Hamiltonian matrix, ${ }^{4}$ and consequently were restricted to relatively small systems, for example, $N \leq 8$ for spin 1 . Recently, there has been significant exploitation of the Lanczös technique $^{5}$ which yields only the first one, two, or three eigenvalues of largest absolute magnitude but, in compensation,
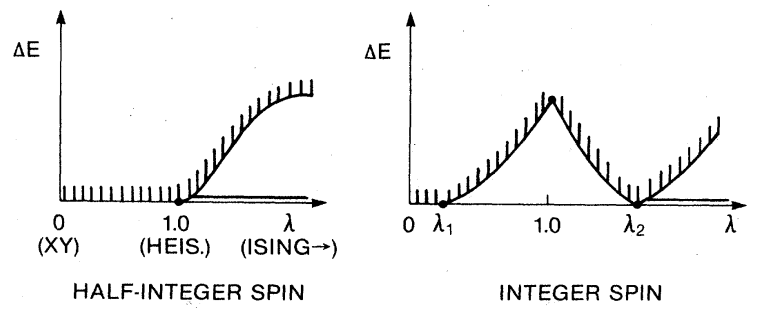

FIG. 1. Low-lying excitations (schematic) as a function of anisotropy $\lambda$ for $X X Z$ Heisenberg spin chains, according to predictions of Haldane. 
can treat longer spin chains. This computational tool applied in conjunction with finite-size scaling techniques has resulted in $T=0$ phase diagrams for $X X Z$ chains with, say, $S=1$ and $S=\frac{3}{2}$, which are consistent with the Haldane picture. ${ }^{6}$ However, equivalent finite-size scaling techniques applied to $S=\frac{1}{2} \quad X X Z$ chains give results which are inconsistent with the exact analytic solution, as noted by Bonner and Müller, ${ }^{7}$ and others. ${ }^{8}$ Hence existing numerical evidence in support of the Haldane conjecture cannot be regarded as conclusive. In fact, a number of independent but essentially similar studies have now appeared $^{6,8-10}$ which contain considerable differences in interpretation by the various authors. The validity of the Haldane conjecture therefore remains controversial and of great interest.

Recently, a new approach to the problem of the lowtemperature static and dynamic correlation functions of quantum-spin chains has been developed by Müller et al. ${ }^{11-13}$ For spin $\frac{1}{2}$ many novel phenomena have been observed, particularly for the case of nonzero applied field, which are strikingly different from what might be expected on the basis of classical spin-wave theory. These quantum effects include the importance of spin-wave double continua (SWDC) for both the longitudinal, $S^{z z}(q, \omega)$, and transverse, $S^{x x}(q, \omega)$, dynamical correlation functions and, in particular, the presence of a soft mode in both $S^{z z}(q, \omega)$ and $S^{x x}(q, \omega)$ which tracks across the Brillouin zone as the field increases from zero to the antiferromagnetic saturation field $H_{s} \cdot{ }^{14,15}$ The differences between quantum (spin- $\frac{1}{2}$ ) low-temperature dynamical behavior and the semiclassical predictions are so pronounced as to invalidate spin-wave theory as a description of spin- $\frac{1}{2}$ quantum chains at low temperatures. This result has not been fully appreciated. The apparent similarity between the $(H=0, \lambda=1)$ dispersion relation of des Cloizeaux and Pearson, ${ }^{16} \Delta E=(\pi / 2) J|\sin q|$ (for $S=\frac{1}{2}$ ), and the Anderson $^{17}$ spin-wave dispersion relation, $\Delta E=J|\sin q|$ (for $S=\infty$ ), has suggested that spin-wave theory might not provide too poor a description even of a onedimensional (1D) Heisenberg magnet in the extreme quantum limit. This is misleading, since recent studies have demonstrated that the des Cloizeaux-Pearson ${ }^{18}$ singlebranch dispersion curve is just the lower bound of a triplet spin-wave double continuum (SWDC), all states of which have nonzero spectral weight and hence contribute to the $T=0$ dynamics. For $H>0$ the presence of several SWDC makes a classical spin-wave description even more inappropriate. ${ }^{11}$ A further example of the deficiencies of spin-wave theory for quantum-spin chains occurs in the case of the biaxially anisotropic $X Y$ chain. An exact analytic solution is possible for this model at a special field (called the Néel field) which depends on the anisotropy. Along the Néel line in field-anisotropy parameter space, quantum effects disappear and the ground state is a fully ordered, two-sublattice, classical Néel state for all values of the spin. ${ }^{19}$ However, exact analytic results for the dynamics at $T=0$ (Ref. 20) show a decidedly nonclassical character in a model where a classical spin-wave approach might be expected to provide an accurate description.

The possibility of an intriguing richness in the static behavior suggested by the Haldane conjecture, and the novel quantum effects apparent in the spin $\frac{1}{2}$ dynamics only highlight the fact that spin chains of general spin $S$ represent nontrivial examples of interacting many-body systems whose detailed properties are of fundamental interest. For example, in the case of Heisenberg chains, the spin- $\frac{1}{2}$ Heisenberg model has exactly integrable character and therefore can, in principle, be solved by Bethe-ansatz techniques. $^{21}$ Heisenberg chains with $\frac{1}{2}<S<\infty$ are believed not to have this property, and at present no exact techniques for their solution are available. Reliance has to be placed on numerical approaches. However, there has recently been a surge of interest in devising and solving 1D quantum systems of higher $\operatorname{spin}\left(>\frac{1}{2}\right)$ which are exactly integrable. The Lai-Sutherland spin- 1 model ${ }^{22}$ appears to constitute the first such example. More recently, Babujian $^{23}$ and Takhtajan ${ }^{24}$ have solved a related model for general spin, and $S_{0} o^{25}$ has solved a spin- $S$ model which is essentially a generalized, spin-anisotropic ( $X X Z$ like) version of the Takhtajan-Babujian isotropic model. A primary motivation of Sogo was to examine the validity of the Haldane conjecture, which fails for his model. However, it appears that for higher-spin models to remain exactly integrable, higher-order terms than simple Heisenberg bilinear exchange have to be introduced, and it is apparent that these additional terms can change the character of the model quite drastically. Therefore the status of the Haldane conjecture is unaffected by the Bethe-ansatz solution of these higher-spin models. Nevertheless, they are very interesting in their own right.

Since higher-spin Heisenberg chains are not at present amenable to analytic solution, it is important to have theoretical insight into approximate techniques. Our technique of choice is a detailed examination of finitesystem calculations on chains as long as possible for a sequence of increasing spin values. A model of interacting quasiparticles introduced by Aghahosseini and Parkinson $^{26}$ has been shown to provide a reasonable description of spin- $\frac{1}{2}$ Heisenberg chains. Our motivation has been to extend this picture to chains with $S>\frac{1}{2}$ to provide more physical insight into this behavior.

In this study we have calculated selected energy eigenvalues, and selected correlation functions using the appropriate eigenfunctions for antiferromagnetic (AFM) Heisenberg spin chains (rings) with periodic boundary conditions. The Hamiltonian matrix has been completely diagonalized only for small systems: For longer chains a Lanczös technique ${ }^{5}$ has been used to determine the two eigenvalues of greatest magnitude for particular constants of the motion. Specifically, these two eigenvalues are the lowest and first excited states for a given value of $S_{T}^{z}=\Sigma_{i} S_{i}^{z}$ and a given value of the wave vector $q$. Previous Lanczös calculations have been concerned only with the zero-field situation $\left(S_{T}^{z}=0\right)$ and generally have considered only the two lowest-lying eigenvalues irrespective of $q$.

As the applied field increases from zero to the AFM saturation (critical) field $H_{s}$, the ground state for finite chains becomes successively an $S_{T}^{z}(S)=0,1,2,3, \ldots, N / 2$ state. Hence by considering the lowest states for all $S_{T}^{z}$ 
values, we are effectively considering the Heisenberg AFM chain in a nonzero field. Recent studies on chains of higher $\operatorname{spin}^{8-10,27}$ have recognized the importance of enlarging the parameter space in order to examine the phase behavior of the system in the vicinity of the Heisenberg AFM point. Such parameters have included spin anisotropy ( $X X Z$-type) and single-ion anisotropy $\left[D \sum_{i}\left(S_{i}^{z}\right)^{2}\right]$. We do not consider here spin and single-ion anisotropy since we are interested in linear Heisenberg antiferromagnets. Our studies are, however, the first to consider magnetic field as an additional parameter. Furthermore, a thorough understanding of the spectral excitations, from which we can infer both static and dynamic properties, is dependent on detailed information with respect to wave vector $q$. For example, of studies cited in Refs. 8-10 and 27, of energy-gap behavior related to the Haldane conjecture, only Sólyom and $\mathrm{Ziman}^{8}$ fully recognize the importance of examining excitations corresponding to various $q$ values, and their studies are restricted to $q=0$ and $\pi$. Our detailed knowledge of eigenstates as a function of $q$ allows a more thorough investigation of the Haldane conjecture. In addition, the dispersion spectra in a field are not only interesting in their own right, but allow us to infer to what extent the novel, nonclassical, features observed in the spin- $\frac{1}{2}$ limit persist with increasing spin value.

Our conclusions from this study are that the additional information on excitation energy gaps remains consistent with the earlier, more restricted, approaches and hence does not give conclusive evidence for the validity of the Haldane conjecture. However, our data do strengthen, by extension, the earlier extrapolative work. Hence the major result of this study is the apparent discovery of the mechanism by which the static and dynamic properties "crossover" from the spin- $\frac{1}{2}$ quantum to the classical limit. Previous authors have studied this problem in the zerofield limit. ${ }^{13,28,60}$ Our finding is that the application of a magnetic field is crucial to an understanding of the mechanism, which is rather complicated. Early work based essentially on a semiclassical perturbation expansion in powers of $1 / S$ may lack validity on two counts. First, the Haldane conjecture indicates that system properties do not vary smoothly with $S$ but depend on whether the spin $S$ is integral or half-integral. Second, the complex phenomena established in this study show conclusively that a $1 / S$ expansion is far too simple a way to treat classical-quantum crossover behavior in spin chains and, by inference, in magnetic systems generally.

The plan of the paper is as follows. In Sec. II we discuss the Haldane conjecture and its implication for the behavior of the magnetization curves as a function of field. The $T=0$ curves of magnetization versus field are examined for $S=\frac{1}{2}, 1, \frac{3}{2}$, and 2 and comparisons are made with the classical, $S=\infty$, curve. Section III is devoted to a discussion of the behavior of the real-space correlation functions $\left\langle S_{i}^{z} S_{i+R}^{z}\right\rangle$ and their Fourier transforms in $q$ space (essentially the integrated intensity, a quantity which can be measured experimentally by neutron scattering techniques). Section IV deals with very interesting phenomena observable in the finite-chain dispersion spectra and their implications for the thermodynamic limit. Section V summarizes our major observations and conclusions.

\section{MAGNETIZATION CURVES AND THE HALDANE CONJECTURE}

In order to elucidate the several features of major interest noted in the Introduction we have undertaken the most comprehensive program of computation extant on eigenvalues and correlation functions of Heisenberg spin chains. For the case of spin $\frac{1}{2}$ our calculations extend out to rings of $N=20$, inclusive, using Lanczös techniques augmented by finite-system Bethe-ansatz analytic calculations. For spin 1 we have extensive information for the purpose of our study on systems of up to 14 spins, inclusive, of up to 10 spins for spin $\frac{3}{2}$, and up to 8 spins for $S=2 .^{29}$ The Lanczös method adopted yields the two lowest eigenvalues and eigenvectors for a given $S_{T}^{z}$ and given $q$, and in order to obtain results on chains of the lengths given above symmetry properties of the Hamiltonian [Eq. (1) with $\lambda=1$ ] were employed. In particular, a technique invented by Blöte involving essentially a combination of reflection and translation operators ${ }^{30}$ was used to avoid the complex matrices encountered in other symmetry-based matrix reduction schemes.

The addition of a Zeeman energy term of the form $g \mu_{B} H \sum_{i} S_{i}^{z}$ to Eq. (1) depresses levels of higher $S_{T}^{z}$ below those of lower $S_{T}^{z}$, and the ground state becomes successively a singlet, triplet, quintet, septet, etc. as the lowest levels for a given $S_{T}^{z}$ cross. The field at which the state of maximal $S_{T}^{z}=N S$ crosses the state with $S_{T}^{z}=N S-1$ is the antiferromagnetic saturation (critical) field $H_{s}$. The state of maximal $S_{T}^{z}$ is, in fact, a component of the fully aligned FM ground state. At $H_{s}$ the magnetization per spin $M$ (strictly $M / N g \mu_{B}$ ) takes on the saturation value $M_{s}=S$ (for $T=0$ ) and thereafter remains constant for $H>H_{s}$. At each crossing point of levels of differing $S_{T}^{z}$, the magnetization per spin (at $T=0$ ) increases discontinuously by an amount $\Delta M=1 / N$ for all $S$, and the result is a step function with a "top step" at saturation magnetization $M_{s}$ and field $H_{s}$, where $H_{s}$ is given by the expression $H_{s}=4 J S / g \mu_{B}$ for all $N$ ( $N$ even).

The behavior of the $T=0$ magnetization curves versus field for 1D antiferromagnets has already been studied in the context of application to real, quasi-1D experimental systems (in a reference of rather restricted access ${ }^{31}$ ). The appearance of the Haldane conjecture, however, indicates that the question should be reexamined with the aid of our longer spin chains. Also, the previous work is already indicative of an unusual, nonuniform approach to the classical limit. For $S<\infty$ the magnetization isotherms near saturation approach the saturation value $M_{s}$ quadratically in field, i.e.,

$$
\left(M_{s}-M\right)^{2} \propto\left(H_{s}-H\right),
$$

whereas the magnetization curve increases linearly in field from 0 to $M_{s}$ in the limit $S=\infty$. (As will become apparent subsequently, the Haldane conjecture would affect the behavior of the magnetization curves only at low fields, not in the vicinity of the saturation field.)

Another reason to study the magnetization curves arises 
from the interacting quasiparticle model of Aghahosseini and Parkinson ${ }^{26}$ for spin $\frac{1}{2}$. The quasiparticles of this model are essentially the quantum equivalents of singlespin deviations (reversed spins) in the classical Ising picture. It is well known that in the case of spin $\frac{1}{2}$, two spin deviations cannot occur at the same site. Aghahosseini and Parkinson ${ }^{26}$ therefore addressed the question of the probability distribution of, say, $r$ reversed spins in terms of a model of quasiparticles interacting through an effective potential which is attractive for ferromagnetic (FM) spin chains and repulsive for antiferromagnetic (AFM) interactions. In an AFM system the effective respulsive interaction induces an equally spaced distribution of the $r$ quasiparticles (or, loosely, the $r$ spin deviations) around the ring.

Calculations based on this model yield remarkably good approximations to the dispersion spectra, even in the lowfield limit, and particularly to the magnetization curve. For spin $>\frac{1}{2}$ the interesting possibility arises of having two spin deviations on one site, and finite-chain calculations allow the evaluation of a quasiparticle model for this situation. In turn, a successful model would provide physical insight into the behavior of chains of higher spin. As it turned out, the quasiparticle model was more informative in the context of the correlation functions, as will be discussed in the next section.

In Fig. 2 magnetization curves as a function of field for periodic systems of $N=12$ spins are presented for the cases $S=\frac{1}{2}$ and $S=1$. In the case of spin $\frac{1}{2}$ it is known $^{32}$ that a smooth curve may be drawn through the horizontal and vertical midpoints of the steps which for a ring as large as 12 spins forms an excellent approximation to the exact magnetization curve. ${ }^{33}$ The argument rests on the fact that both height and width of the steps of the magnetization step function shrink in the thermodynamic limit as $N \rightarrow \infty$ to give a smooth curve. Since the argument has proved to be valid for $S=\frac{1}{2}$, it is reasonable to presume its validity for $S>\frac{1}{2}$ also. Hence, in Fig. 2,

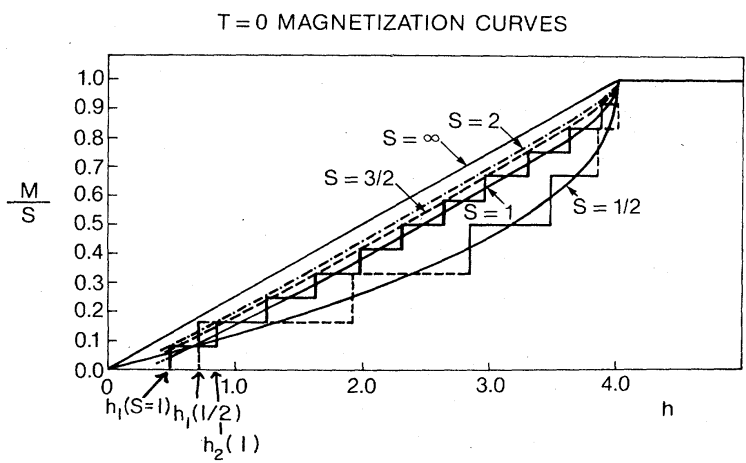

FIG. 2. Extrapolated $T=0$ magnetization isotherms for Heisenberg antiferromagnetic chains of various spin value. Uncertainty over the validity of the Haldane conjecture is reflected in the fact that the extrapolations are not completed in the region of low field. Portions of the step-function curve corresponding to $S=\frac{1}{2}, N=12$ are shown dashed where confusion might result with the superimposed step-function curve for $S=1, N=12$. smooth "midpoint" functions are shown for both $S=\frac{1}{2}$ and $S=1$. Also shown, for comparative purposes, is the linear curve for $S=\infty$. It is immediately apparent that the midpoint curve for spin 1 is notable for linear character over much of its extent, in contrast to the curve for spin $\frac{1}{2}$, which shows marked curvature for $M \geq \frac{1}{2} M_{s}$. In this respect, the spin-1 curve much more closely resembles the classical curve. As $M$ increases, curvature eventually sets in for spin 1 as saturation is approached, which appears to have the same quadratic behavior as for spin $\frac{1}{2}$. In Fig. 2 also, midpoint curves are shown for $S=\frac{3}{2}$, $N=10$ and $S=2, N=8$, the longest rings available for these spin values. The linear character becomes more pronounced as $S$ increases and the region of curvature near saturation decreases, indicating the nature of quantumclassical crossover for the $T=0$ magnetization curve. In the $S=\infty$ limit the region of curvature disappears and the curve is linear over the entire regime. However, noting that the saturation field is given by $g \mu_{B} H_{s} / J=4 S$, it is convenient to plot the curves of Fig. 2 in terms of a spin-reduced field, $h=g \mu_{B} H / J S$, for convenient comparison of curves of different $S$. In a plot versus unreduced field $H$, the size of the quadratic region remains roughly constant, independent of $S$.

We mentioned that, in general, analytic Bethe-ansatz techniques cannot be used for Heisenberg chains with $S>\frac{1}{2}$. However, it is possible to use a Bethe-ansatz approach for the problem of one-spin deviation (trivial), and also two-spin deviations ${ }^{34}$ for a rather wide variety of spin Hamiltonians in the thermodynamic limit. The form of the magnetization curve as $M \rightarrow M_{s}$ is, in fact, directly related to the problem of two reversed spins and hence may be obtained analytically. For spin $\frac{1}{2}$ an expression obtained by perturbation theory and subsequently by a Bethe-ansatz approach is ${ }^{32}$

$$
M / M_{s}=1-\frac{4}{\pi}\left(1-H / H_{s}\right)^{1 / 2}
$$

For general spin our exact expression is

$$
M / M_{s}=1-A(S)\left(1-H / H_{s}\right)^{1 / 2},
$$

where $A(S)=2 / \pi S$.

Let us now analyze the magnetization curves of Fig. 2 from another viewpoint. The behavior of the curves for very low fields relates directly to the Haldane conjecture. The distance from zero to the first step $\left(0-h_{1}\right.$ in Fig. 2) directly measures the finite-chain energy gap between the singlet ground state and first excited triplet. The Haldane conjecture discussed in the Introduction implies that a nonzero excitation gap persists in the thermodynamic limit for integer-spin Heisenberg chains. The singlet-triplet energy gap for spin $\frac{1}{2}$, plotted as a function of $1 / N$, tends to zero as $N \rightarrow \infty$, consistent with the analytic solution which yields a gapless excitation spectrum. A similar $1 / N$ plot for the elementary excitations for spin-1, spin$\frac{3}{2}$, and spin-2 Heisenberg chains, which also turn out to be singlet-triplet energy gaps, is presented as Fig. 3. Our calculations extend and check calculations of Kolb, Botet, and Jullien, ${ }^{6}$ and similar conclusions can be drawn. ${ }^{35}$ For the case of $\operatorname{spin} \frac{1}{2}$ the $1 / N$ plot is not straight but shows 


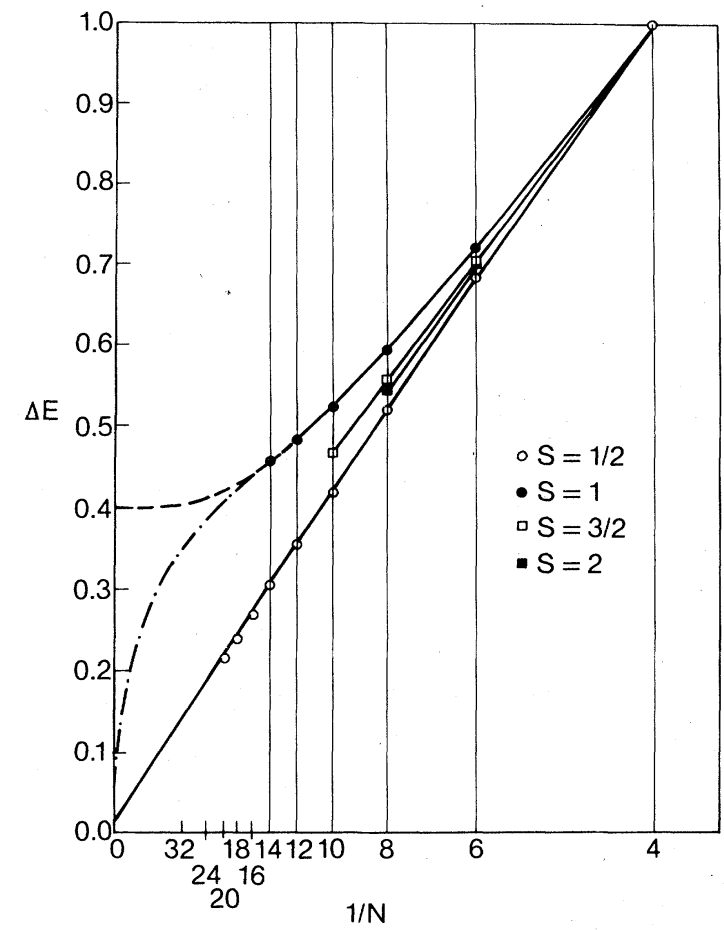

FIG. 3. Plot of the singlet-triplet "primary" excitation gaps for various spin values. In the case of spin 1 the dashed line represents an extrapolation which tends to a nonzero Haldane gap asymptotically with $1 / N$ and $N \rightarrow \infty$. The dot-dashed curve represents a more complicated form of asymptotic, large$N$ behavior which cannot be conclusively ruled out on the basis of data up to $N=14$ only.

some (concave downwards) curvature introducing some uncertainty in the extrapolation, but the trend is clearly consistent with a value zero (no gap) in the $N \rightarrow \infty$ limit. For the case of spin 1, however, a dramatic difference occurs. Curvature appears in the $1 / N$ plot which is concave upwards, and furthermore increases with increasing $N$, strongly indicating a finite nonzero gap in the $N \rightarrow \infty$ limit consistent with the Haldane conjecture. Hence the behavior of the plot for spin $\frac{3}{2}$ is particularly significant. The small $N$ values $(4,6,8)$ show the same upward curvature as the corresponding points for spin 1 , indicating a nonzero gap in the limit, but the point for largest $N$ (=10) reverses this trend. A smooth curve drawn through the four points for spin $\frac{3}{2}$ would have an inflection point before a down-turn sets in, indicating the possibility of a zero value in the limit. Hence this particular set of finite-chain results is consistent with the Haldane conjecture. Except for the possibility of an essential singularity, these extrapolations together with the form of the $T=0$ magnetization curve would provide convincing support for the conjecture. However, the existence of an "inflection point" for $S=\frac{3}{2}$ reflecting a change in trend with increasing $N$ is an indication, presumably, of irregular convergence resulting from the presence of an essential singularity, Bonner and Müller ${ }^{7}$ estimated that for $S=\frac{1}{2}$, chains as long as 25 spins would be required for the asymptotic large- $N$ behavior to become apparent. This estimate is supported by our Bethe-ansatz calculations up to $N=20$ spins, inclusive. The possibility cannot be ruled out that for sufficiently large $N(N>12)$ an "inflection point" and ultimate trend of the extrapolation to zero gap may occur for $S=1$ as it does for $S=\frac{3}{2}$.

However, there is another possibility to be considered. The gap discussed above is the energy gap between the AFM ground state, which is always a singlet state located at $q=0$ and the lowest-lying triplet $S_{T}\left(S_{T}^{z}\right)=1$ state, which lies at $q=\pi$. An examination of a plot of the lowest-lying excitations for various $S_{T}\left(S_{T}^{z}\right)$ values for a given value of $q$ reveals other energy gaps which might possibly extrapolate to zero even if the singlet-triplet gap does not. These other possibilities are referred to for convenience as "higher gaps" and include a singlet-quintet $\left(S_{T}=0,2\right)$ and singlet-septet $\left(S_{T}=0,3\right)$ gap. There is a low-lying excited singlet state at $q=\pi$, and hence we consider also a singlet-singlet gap. For the case of $S=1$, these gaps are shown in Fig. 4 as a function of $1 / N$. If the singlet-quintet $\Delta(0,2)$, singlet-septet $\Delta(0,3)$, and singlet-singlet $\Delta(0,0)$ gaps are normalized ${ }^{36}$ by factors of 3,6 , and 4, respectively, all take the same value for $N=4$. The results are interesting. All gaps show a trend to a nonzero value in the $N \rightarrow \infty$ limit. However, the curvature of the $\Delta(0,1)$ gap is monotonically increasing with $N$ and concave upward. A similar trend occurs also for the $\Delta(0,2)$ gap although the curvature is definitely less pronounced. For the $\Delta(0,3)$ gap, however, the curvature is concave downwards out to $N \sim 10$, but then reverses, indi-

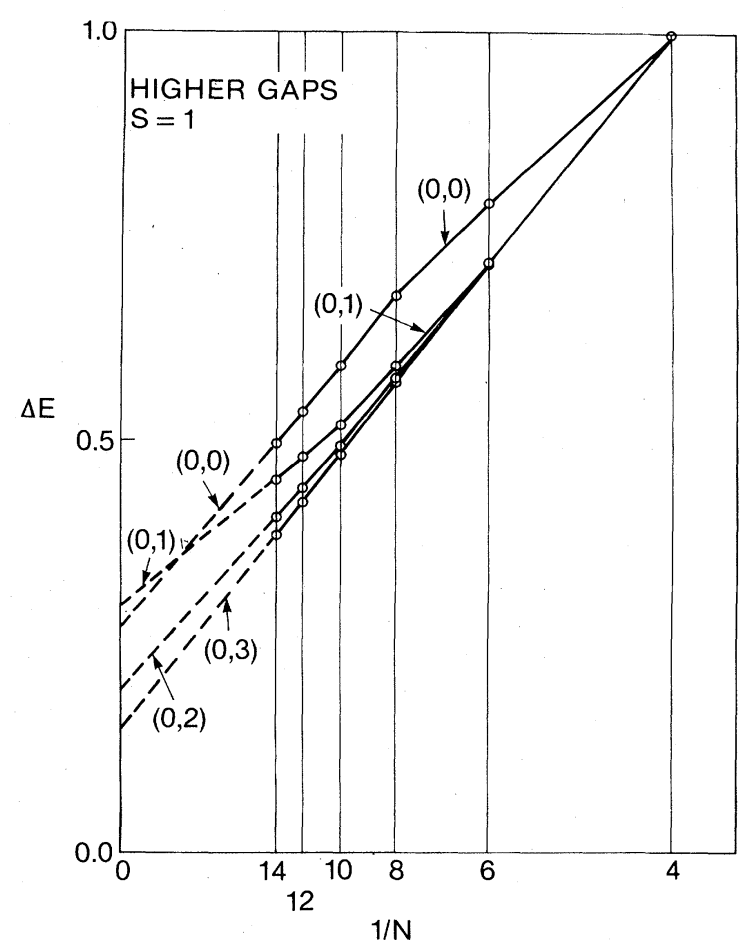

FIG. 4. Plot of "higher" excitation energy gaps, i.e., gaps between singlet ground-state and the lowest-lying $S_{T}=1,2,3,0$ excitations for spin 1 , versus $1 / N$. All gaps show a trend to a nonzero value in the $N \rightarrow \infty$ limit. 
cating a nonzero value in the $N \rightarrow \infty$ limit. A similar tendency occurs also for the $(0,0)$ gap, and the tendency is more pronounced, as is apparent from Fig. 4. The possibility cannot be discounted that the irregular convergence associated with the "primary" gaps for $S=1, \frac{3}{2}$, and 2 , and with the "higher gaps" for $S=1$ is a manifestation of a singularity at the Heisenberg point, as suggested by Bonner and Müller. ${ }^{7}$

Some of the higher gaps can be directly related to the magnetization curves of Fig. $2 .{ }^{37}$ Let us denote the locations of the first, second, and third magnetization steps by $h_{1}, h_{2}$, and $h_{3}$, respectively. There is an interesting and striking difference between the magnetization isotherms for $S=\frac{1}{2}$, on the one hand, and $S=1, \frac{3}{2}$, and 2 on the other. By inspection, for $S=\frac{1}{2}$, there is an approximate relation

$$
h_{2} \sim 3 h_{1} \text { and } h_{3} \sim 5 h_{1},
$$

but

$h_{1}=E_{1}-E_{0}, \quad h_{2}=E_{2}-E_{1}$, and $h_{3}=E_{3}-E_{2}$.

Hence

$$
\begin{aligned}
& h_{1}=E_{1}-E_{0}=\Delta(0,1), \\
& h_{1}+h_{2}=E_{2}-E_{0}=\Delta(0,2), \\
& h_{1}+h_{2}+h_{3}=E_{3}-E_{0}=\Delta(0,3),
\end{aligned}
$$

and substituting from (5), we have

$$
\Delta(0,2)=4 \Delta(0,1) \text { and } \Delta(0,3)=9 \Delta(0,1) .
$$

Equation (8) gives the appropriate normalizations for the case of spin $\frac{1}{2}$. The geometrical significance of $(8)$ is that a straight line through the midpoints of the first two steps will pass through the origin, in accordance with the exact solution, i.e., the distance from zero field to the first step is half the distance between first and second steps.

The surprising feature for $S>\frac{1}{2}$ is that the distance from zero field to the first step is at least equal to the distance between first and second steps, a feature which is consistent with a Haldane gap, even for spin $\frac{3}{2}$ ! Hence there is an approximate relation

$$
h_{2} \sim 2 h_{1} \text { and } h_{3} \sim 3 h_{1},
$$

from which we may deduce that

$$
\Delta(0,2)=3 \Delta(0,1) \text { and } \Delta(0,3)=6 \Delta(0,1) .
$$

Equation (10) gives the normalization factors used in Fig. 4. Note that relations (9) and (10) are exact for $N=4 .^{38}$ These observations based on the $T=0$ magnetization curves suggest an alternative possibility to the Haldane conjecture: that $S=\frac{1}{2}$ is a very special case, and that chains with $S>\frac{1}{2}$ are more similar to one another than to spin $\frac{1}{2}$. More evidence for this possibility will appear in our subsequent discussions of the behavior of correlation functions and dispersion spectra.

Appendix A comprises a set of tables for the longest spin chains for a given spin value (Tables $\mathrm{I}-\mathrm{V}$ ). These tables list the two lowest eigenvalues for all allowed values of $S_{T}^{z}$ and all values of wave vector $q$. Finite-chain $T=0$ magnetization curves are constructed from a knowledge of the lowest eigenvalue for a given $S_{T}^{z}$ irrespective of $q$.

\section{LONGITUDINAL CORRELATION FUNCTIONS}

Very little exact information is at present available concerning the static and dynamic spin-pair correlation functions of Heisenberg spin chains, even for spin $\frac{1}{2}$. For the case of spin $\frac{1}{2}$, some analytic results derived using Betheansatz techniques are available for the low-temperature thermodynamic properties, but information on the thermodynamic properties for general temperature is derived from numerical studies. Since the behavior of the correlation functions is even less understood, their study is important.

The static zero-field spin-spin correlation function in real space is given by $\left\langle S_{i}^{\mu} S_{i+R}^{\mu}\right\rangle$, where $\mu=x, y, z$, representing the average correlation between two spins $R$ lattice sites apart. Its Fourier transform in $q$ space is given by

$$
\begin{aligned}
S(q)= & \sum_{R} e^{-i q R}\left\langle S_{i}^{\mu} S_{i+R}^{\mu}\right\rangle \\
& \text { with } q=2 \pi r / N, r=0,1, \ldots,(N-1) .
\end{aligned}
$$

The time-dependent correlation function is given by $\left\langle S_{i}^{\mu}(t) S_{i+R}^{\mu}(0)\right\rangle$, representing the interaction of a spin at time $t$ with a second spin at time zero, $R$ lattice sites away. The Fourier transform is the dynamical correlation function (or structure factor) given by

$$
S^{\mu \mu}(q, \omega)=\sum_{R} e^{-i q R} \int_{-\infty}^{\infty} d t e^{i \omega t}\left\langle S_{l}^{\mu}(t) S_{i+R}^{\mu}(0)\right\rangle
$$

In this work we employ periodic boundary conditions, in which case the correlation function $\left\langle S_{i}^{\mu} S_{i+R}^{\mu}\right\rangle$ is independent of the location of site $i$ on the ring.

Some exact results are available for spin- $\frac{1}{2}$ correlations of AFM Heisenberg chains in the ground state (i.e., at $T=0$ ) in the thermodynamic limit. The value for the nearest-neighbor correlation function $\left\langle S_{i}^{\mu} S_{i+1}^{\mu}\right\rangle$ is known exactly since it is related to the ground-state energy determined by Hulthén ${ }^{39}$ using Bethe-ansatz techniques. Takahashi $^{40}$ obtained an exact result for the secondneighbor correlation function at $T=0,\left\langle S_{i}^{\mu} S_{i+2}^{\mu}\right\rangle$. Exact analytic work of $\mathrm{Baxter}^{41}$ on the staggered spontaneous polarization of the antiferroelectric $F$ model implies that the long range order, defined as $\lim _{R \rightarrow \infty}\left\langle S_{i}^{\mu} S_{i+R}^{\mu}\right\rangle$, must vanish for the linear Heisenberg AFM at $T=0 .{ }^{42}$ Earlier numerical work of Bonner and Fisher ${ }^{32}$ using finite-chain extrapolations correctly predicted vanishing of the longrange order (LRO), and yielded a value for the secondneighbor correlation in agreement with Takahashi's exact result ${ }^{40}$ to within $3 \%$, giving confidence in the reliability of finite-chain extrapolations, at least for spin $\frac{1}{2}$. The Bonner-Fisher calculations extended to rings of $N=10$ spins, and also included values for third- and fourthneighbor correlations. Very recently Grieger ${ }^{43}$ has found exact values for correlations $\left\langle S_{i}^{\mu} S_{i+R}^{\mu}\right\rangle$, where 
$R=1,2, \ldots, N / 2$, for even- $N$ rings of up to $N=16$, inclusive, using the Bethe-ansatz approach. Independently and contemporaneously, Borysowicz, Kaplan, and Horsch have performed parallel calculations using both Betheansatz and Lanczös techniques on periodic systems up to 16 spins, inclusive, and also on free-ended chains using Lanczös techniques. ${ }^{44}$ Other approximate calculations of linear Heisenberg AFM correlations in the ground state include work of Luther and Peschel using a Luttingermodel approach ${ }^{45}$ and Monte Carlo simulations of Hirsch et al. for $N$ up to $100 .{ }^{46}$ However, it is now known that a Luttinger-model approach will not reveal logarithmic corrections in the approach to the thermodynamic limit with system size $N$, or in the behavior of the correlations in the thermodynamic limit as a function of $R .{ }^{47}$ Hirsch et $a l .{ }^{46}$ were actually interested in the Fourier transform of the finite-system correlations at $q=\pi\left[S_{N}(\pi)\right]$ and the approach with increasing $N$ to the thermodynamic limit. Horsch and Kaplan noted that the exact results for $N$ through 10 (Refs. 32 and 48) were in disagreement with the values obtained by Monte Carlo methods by an amount lying well outside the quoted statistical errors. Hence it must be concluded that these two other approximate methods, Monte Carlo and Luttinger techniques, do not supersede finite-chain calculations, which remain the most trusted and informative approach to this problem.

The above discussion is concerned with the behavior of the static correlations at $T=0$ and in zero applied field. Investigations of the static correlation functions in an applied field have been made by Aghahosseini and Parkinson $^{26}$ for spin- $\frac{1}{2}$ Heisenberg chains, and by Haldane, ${ }^{49}$ who considered the general spin- $\frac{1}{2} X X Z$ model in a Luttinger format. When the applied field $H$ is no longer zero a distinction must be made between the longitudinal correlation functions $\left\langle S_{i}^{z} S_{i+R}^{z}\right\rangle$ and the transverse correlations $\left\langle S_{i}^{x} S_{i+R}^{x}\right\rangle$, more conveniently expressed in terms of the operators $S^{ \pm}$as $\left\langle S_{i}^{+} S_{i+R}^{-}\right\rangle$and $\left\langle S_{i}^{-} S_{i+R}^{+}\right\rangle$. Aghahosseini and Parkinson ${ }^{26}$ studied numerically both longitudinal and transverse correlations for periodic Heisenberg chains up to $N=12$ spins as a function of magnetization. As is well known, ${ }^{32}$ in zero field the correlations $\left\langle S_{i}^{\mu} S_{i+R}^{\mu}\right\rangle$ alternate in sign according to whether $R$ is odd or even. Aghahosseini and Parkinson ${ }^{26}$ observed that the transverse correlation retained this alternating (AFM) character essentially unchanged for all fields up to the saturation field $H_{s}$. The longitudinal correlation, however, changed considerably, in a manner which suggested a reflection in real space of a periodicity in $q$ space first discovered in a Hartree-Fock approximate calculation by Pytte $^{50}$ and studied analytically by Ishimura and Shiba ${ }^{51}$ and Aghahosseini and Parkinson. ${ }^{52}$ It was pointed out ${ }^{52}$ that the calculated correlation functions were consistent neither with a classical spiral ground-state spin structure nor with the two-sublattice spin-flop model of Wang and Callen. ${ }^{53}$. In this work the longitudinal correlations are analyzed in detail for longer chains for spin $\frac{1}{2}$, and also for chains with $S>\frac{1}{2}$. (The computation of the transverse correlations is considerably more difficult and has not been attempted.) To the best of our knowledge, these are the first static correlation function calculations for Heisenberg chains with $S>\frac{1}{2}$, and the re- sults show striking differences from the results for $S=\frac{1}{2} .54$

Concerning the dynamical correlation functions of AFM Heisenberg chains with spin $\frac{1}{2}$ at low temperature, finite-chain calculations have been made by Müller et al. ${ }^{11}$ in zero field, and by Müller et al. ${ }^{11}$ and Ishimura and Shiba $^{55}$ for nonzero field. The work of Müller et al. ${ }^{11}$ in particular, demonstrates that several unusual phenomena are to be expected which differ strikingly from classical spin-wave predictions. These quantum effects include (a) the importance of multiple spin-wave double continua in both longitudinal $S^{z z}(q, \omega)$ and transverse $S^{x x}(q, \omega)$ dynamical correlation functions, (b) the expected presence of multiple structure in $S^{z z}(q, \omega)$ and $S^{x x}(q, \omega)$ as a consequence of point (a), and, in particular, (c) the presence of a soft mode in both $S^{z z}(q, \omega)$ and $S^{x x}(q, \omega)$ which tracks across the Brillouin zone as the field increases from zero to the AFM saturation field $H_{S}$. While it would be of great interest to examine the dynamical correlation functions for $S>\frac{1}{2}$ in as much detail, the difficulty of such a project is at present prohibitive, since large numbers of eigenstates are involved even for a $T=0$ calculation. However, the striking soft-mode feature is apparent in the static correlations (and also in the dispersion spectra, as we shall observe in the next section). Hence our calculations can give information on the effect of higher spin on the moving soft mode.

We shall first study the field-dependent spin- $\frac{1}{2}$ correlations in real space, and then make appropriate comparisons with higher-spin correlations. Questions of interest in connection with higher spin are the behavior of the LRO correlation function, the behavior of the static correlation function, which is an uninteresting constant in the case of spin $\frac{1}{2}$, and the relevance of the AghahosseiniParkinson spin-deviation quasiparticle model to higherspin systems. Finally, we shall relate the behavior of the Fourier transform $S^{z z}(q)$ to features observed in the realspace correlations.

Extrapolated estimates for the various longitudinal correlation functions for spin $\frac{1}{2}$ are shown in Fig. 5, plot-

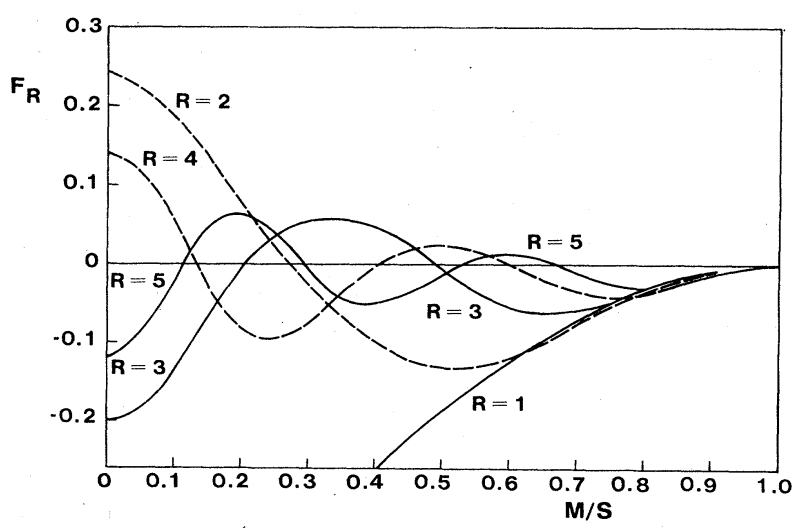

FIG. 5. Extrapolated behavior of the longitudinal spin- $\frac{1}{2}$ correlations $\left\langle S_{i}^{z} S_{i+R}^{z}\right\rangle$ showing the pronounced oscillatory character as a function of magnetization (or field) which is a reflection of the soft mode in the dispersion spectra. For convenience, extrapolations for $R$ even are shown dashed. 
ted as a function of normalized magnetization per spin $M / S$. In the presence of a nonzero magnetic field, the appropriate correlation functions are not the functions $\left\langle S_{i}^{z} S_{i+R}^{z}\right\rangle$ but functions which represent fluctuations about the mean magnetization. Specifically, the finitechain correlation functions are defined as $F_{R}(N)$, where

$$
F_{R}(N)=\left(\left\langle S_{i}^{z} S_{i+R}^{z}\right\rangle-M^{2}\right) / S^{2},
$$

where $S=\frac{1}{2}$ for the plots of Fig. 5. The extrapolated limiting curves are denoted by $F_{R}(N \rightarrow \infty)=F_{R}$.

Note first that the curves are oscillatory as a function of $M$, with the exception of the nearest-neighbor correlation $F_{R=1}=F_{1}$, which increases monotonically from a minimum negative value (not shown) for $M=0$ to zero as the magnetization reaches saturation, $M / S=M_{s} / S=1.0$. In fact, all the $F_{R}$ vanish at $M_{s}$, since quantum fluctuations disappear in this limit. The oscillatory character is dependent on the value of $R$ : the curve for $R=1$ has one zero (at $M / S=1.0$ ), the curve for $R=2$ has two zeros, and so on. At $M=0$ the values for $F_{1}$ and $F_{2}$ are known exactly as -0.59086 (Ref. 32) and +0.24272 (Ref. 40), respectively. The $M=0$ values for $F_{3}, F_{4}$, and $F_{5}$ are taken from Ref. 43 where extrapolations are made on periodic systems of up to 16 spins. For other values of $M$, points for $N=14,12$, and 10 define a limiting, $N \rightarrow \infty$, curve quite accurately. The exception, perhaps, is $F_{5}$, where the relatively large number of oscillations implies some uncertainty in extrapolation for rings up to 14 spins only, since there are not enough points to trace out the limiting curve very accurately in the vicinity of the maxima and minima, and finite-size effects are accentuated. The fact that the $F_{R}$ are characterized by a number of zeros equal to $R$ implies the well-known result that in zero field $(M=0)$ the correlations oscillate in sign with a multiplying factor $(-1)^{R}$ for both finite and infinite $N$.

It is worth noting that oscillatory character with $M$ occurs also in the case of the out-of-plane $(Z Z)$ correlations for the spin- $\frac{1}{2} X Y$ chain. ${ }^{56}$ The corresponding expression is

$$
\left\langle S_{i}^{z} S_{i+R}^{z}\right\rangle-M_{z}^{2}=4\left[\frac{\sin [R \arccos (h / J)]}{\pi R}\right]^{2},
$$

where $J$ is the exchange constant and $h$ (reduced field) $=g \mu_{B} H$. The saturation field for the $X Y$ model is given by $h / J=1.0$. The magnetization $M_{z}$ is related to $h$ via the expression

$$
M_{z}(h)=\left\{\begin{array}{l}
(1 / \pi) \arcsin (h / J), \quad 0 \leq h \leq h_{s} \\
\frac{1}{2}, \quad h \geq h_{s} .
\end{array}\right.
$$

Hence the functions $F_{R}^{X Y}$ also show oscillatory character as a function of $M$ and $R$, superimposed on a $1 / R^{2}$ algebraic decay reflecting the absence of LRO for $0 \leq h<h_{s}$. Haldane ${ }^{49}$ has also predicted algebraic decay with $R$ for the correlation functions of the linear Heisenberg AFM. However, he predicts nonuniversal behavior in that the decay exponent $\eta_{z}$, defined through an asymptotic algebraic decay, $1 / R^{-1+\eta_{z}}$, is a continuously varying function of $M$, decreasing as $M$ increases to saturation. Hal- dane presents numerical results for $\eta_{z}(M)$ and it would be interesting to attempt to make comparisons. However, the necessary extrapolations are far from straightforward. ${ }^{57}$

A previous numerical analysis has been made of the behavior of the spin- $\frac{1}{2}$ finite-chain longitudinal correlation functions, $F_{R}(N)$, in zero field. ${ }^{32,44}$ Consider a ring of spins with $N$ even. The correlations both alternate in sign and, in general, decrease in magnitude out to $R \sim N / 2$, i.e., out to halfway around the ring. The fact that there are two distinct sequences, $F_{R}(N)>0, R$ even, and $F_{R}(N)<0, R$ odd, means that the decay of $\left|F_{R}(N)\right|$ is not monotonic, and hence it makes sense to take an average of the two sequences. A case in point is the determination of the LRO by extrapolation to the limit $N \rightarrow \infty$. The LRO was defined through the relation

$$
F_{\infty}=\lim _{R \rightarrow \infty}\left|\lim _{N \rightarrow \infty} F_{R}(N)\right| \text {. }
$$

To estimate $F_{\infty}$, therefore, minimum means

$$
F_{\min }(N)=\frac{1}{2}\left[F_{N / 2}(N)-F_{N / 2-1}(N)\right]
$$

were constructed and extrapolated to $N \rightarrow \infty$. A wellbehaved sequence was obtained which extrapolated essentially to zero as a function of $1 / N$. The $N \rightarrow \infty$ prediction was subsequently confirmed by Baxter's ${ }^{41}$ exact result.

Let us now turn to Heisenberg chains with $S>\frac{1}{2}$. The LRO is a quantity of particular interest. A specific prediction of Haldane ${ }^{2}$ is that for integer-spin Heisenberg AFM chains in zero field the ground state is a singlet, and the correlations decay to zero exponentially, rather than as a power law. According to Haldane,

$$
F_{R} \sim A R^{-1 / 2} \exp (-C R)+B R^{-2} \exp (-2 C R),
$$

as $R \rightarrow \infty, A, B$, and $C$ are constants which presumably depend on the particular integer-spin value. Since the class of half-integer-spin chains is expected to behave like spin $\frac{1}{2}$, the LRO is expected to decay to zero as a power law in $R$ (or $N$ ).

It is observable in our finite-chain results that the effect of the two sequences, $R$ odd and even, is significantly reduced for $S>\frac{1}{2}$. Nevertheless, for consistency we again form minimum means and plot versus $1 / N$, as shown in Fig. 6. The points for $S=\frac{1}{2}$ show residual oscillatory effects depending on whether $N / 2$ is odd or even, but clearly demonstrate an overall trend to zero in the $N \rightarrow \infty$ limit. The points for $S=1$ show irregular convergence, but the last three points $(N=12,10,8)$ show a strong trend to zero which may reflect an asymptotic exponential convergence. The points for $S=\frac{3}{2}$ do show downwards curvature, but a trend to zero is not clearly indicated for $N$ up to 10 , while for spin 2 no clear trend toward zero is observed. Since the Haldane conjecture ${ }^{2}$ explicitly predicts that the LRO should vanish, either algebraically or exponentially, as $N \rightarrow \infty$, support is not provided by our higher-spin correlations, at least by this preliminary analysis. Slow convergence with $N$ is apparently a problem here, in which case more detailed analysis may not be fruitful. 


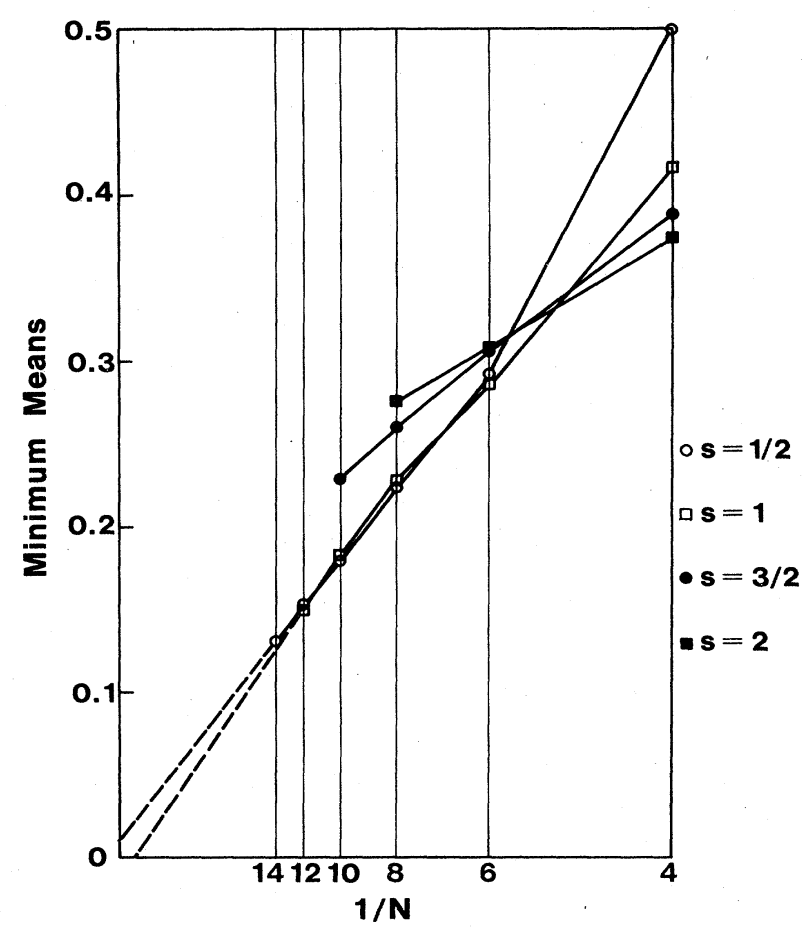

FIG. 6. Plots of "minimum means" versus $1 / N$ for various spin value to examine the spin dependence of the $T=0$ longrange order (LRO). Slow convergence makes interpretation difficult.

Let us now discuss the autocorrelation function, $F_{0}(N)$. For spin $\frac{1}{2}$, the actual autocorrelation function $\left\langle S_{i}^{2}\right\rangle$ takes the constant value of $S^{2}=\frac{1}{4}$, independent of $M$ (or $H$ ), reflecting the fact that each spin $S_{i}$ has only two orientations, $\pm \frac{1}{2}$. For spin 1, however, a single spin $\mathbf{S}_{i}$ has three possible orientations, such that $S_{i}^{z}=+1,0,-1$, and the relative probabilities of the three orientations may be expected to vary with field. Hence the autocorrelation functions $F_{0}(N)$ for $S>\frac{1}{2}$ are observed to show structure. In Fig. 7 the $N \rightarrow \infty$ limiting curve $F_{0}(N \rightarrow \infty)$ is observed to be well defined by the points for $F_{0}(12)$ and $F_{0}(10)$, and is observed to display two inflection points although there may be some uncertainty near $M \rightarrow 0$. In the region near saturation $(M / S \rightarrow 1)$, exact asymptotic results may be derived by Bethe-ansatz techniques for oneand two-spin deviations as a general function of spin $S$. For example, the asymptotic slope of $F_{0}(\infty)$ as $M / S \rightarrow 1$ is $-1 / S$. A one-spin-deviation calculation gives a parabolic expression which is a reasonable description of the spin-1 limiting curve for $1 \geq M / S \geq 0.7$. Defining a parameter $\sigma$ to measure the deviation of the magnetization from saturation, $\sigma=(1-M / S)$, we have

$$
F_{0}(\infty) \sim \sigma / S-\sigma^{2}
$$

and consideration of two-spin deviations extends expression (19) to higher powers of $\sigma$ :

$$
F_{0}(\infty) \sim \sigma / S-\sigma^{2}+\frac{1}{8} \pi^{2} S^{2}(2 S-1)^{2} \sigma^{4}+\cdots
$$

The term in $\sigma$ gives rise to an upturn for increasing $\sigma$,

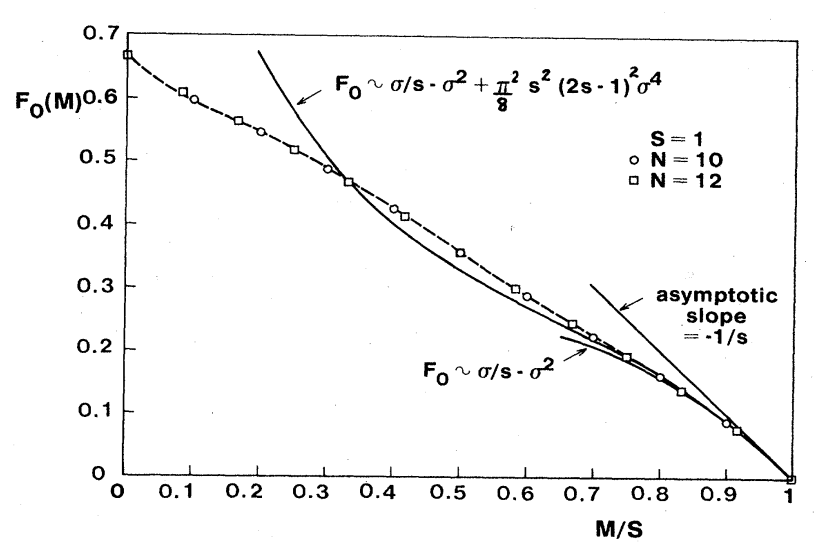

FIG. 7. Plot of the extrapolated autocorrelation function for spin $1, F_{0}(\infty)$, shown dashed, together with points for finite $N$.

thus accounting for the first inflection point observed in the extrapolated curve for $S=1$. However, for $M / S \lesssim 0.5$ a change of curvature occurs which is evidently the result of higher-order deviation processes not taken into account in (20). Finally, for $M / S \lesssim 0.1$ a situation occurs which may indicate a second inflection point, or it may be that the points at $M=0$ are anomalous, relating to the Haldane conjecture. Note that for spin $\frac{1}{2}$, the coefficient of the term in $\sigma^{4}$ in (20) vanishes and (19) gives the exact result. For the case of $S=\frac{3}{2}$, there is less structure apparent in $F_{0}(\infty)$ than for spin 1 and there is only a single inflection point at $M / S \sim 0.4$. Expressions (19) and (20) are in reasonable agreement with the extrapolated curve over a more restricted range of $\sigma$ : namely, for $M / S \gtrsim 0.7$ for expression (20). In fact, the situation is reminiscent of the magnetization curves of Fig. 2, where quantum effects are apparent only in a region $M_{s} \geq M \geq M_{s}(1-1 / 2 S)$.

Since the autocorrelation function effectively measures the probability of an average spin's being in a particular allowed quantum state, the autocorrelation function for $S=1$, say, can be used in conjunction with a knowledge of the magnetization to infer the average spin deviation as a function of magnetization. This information can be used to examine the validity of the AghahosseiniParkinson spin-deviation model for $S>\frac{1}{2}$.

Let $P(+1), P(0)$, and $P(-1)$ denote the probability of the average spin's being in the three allowed quantum states for $S=1$, namely $S^{z}=+1,0$, and -1 , respectively. At saturation, $M / S=1$, the ground state is the fully aligned FM-type state with all spins taking the maximal values $S_{i}^{z}=+1$, and the total spin $S_{T}$ and its $z$ component $S_{T}^{z}$ taking the value $N S=N$. Hence we must have $P(+1)=1, P(0)=P(-1)=0$. In the case of spin $\frac{1}{2}$, the ground state is a state with $S_{T}=S_{T}^{z}=0$ ( $N$ even), which implies a state with $N / 2$ spins "up" and $N / 2$ spins "down", i.e., $P\left(+\frac{1}{2}\right)=P\left(-\frac{1}{2}\right)=\frac{1}{2}$. Assuming a similar situation for $S=1$, we expect $P(+1)=P(0)$ $=P(-1)=\frac{1}{3}$. We can check this since $F_{0}=\frac{2}{3}($ all $N)$. But.

$$
\begin{aligned}
F_{0} & =P(+1) \times(+1)^{2}+P(0) \times 0+P(-1) \times(-1)^{2} \\
& =P(+1)+P(-1)=\frac{2}{3} .
\end{aligned}
$$


Figure 8 is a plot of $P(+1), P(0)$, and $P(-1)$ as a function of magnetization for $N=12$, determined as above. We observe that $P(+1)$ decreases monotonically from its maximum value of +1 at $M=1$ to its value of $\frac{1}{3}$ at $M=0$. The probability $P(0)$ rises from zero at $M=1$, reaches a maximum of $\sim 0.42$ at $M \approx 0.3$, then decreases to $\frac{1}{3}$ at $M=0$. The probability $P(-1)$ rises very slowly initially from zero as $M$ decreases from 1 , becoming significant at $M \sim 0.5$, and then increases rather rapidly to the value $\frac{1}{3}$ at $M=0$. The physical implications are very interesting, especially in terms of the AghahosseiniParkinson spin-deviation model. The function $P(0)$ represents the probability of having a single-spin deviation at a site, and $P(-1)$ represents the probability of having two spin deviations at the same site (a feature which cannot occur for $S=\frac{1}{2}$ ). Observe that since the twospin-deviation probability is very small in the region $M_{s} \geq M \geq M_{s}(1-1 / 2 S)$, a single-spin-deviation picture of the Aghahosseini-Parkinson type should provide a very good description of the physical situation. For the "quantum" regime $M_{s} \geq M \geq 0.5 M_{s}$ the spin-deviation picture is essentially similar to that for $S=\frac{1}{2}$. Only in the "classical" regime $0.5 M_{s} \geq M \geq 0$ do the effects of having two spin deviations at the same site become appreciable. Unfortunately, we cannot test this interesting result for $S>1$, since additional information beyond that supplied by a knowledge of the autocorrelation function and magnetization is required.

Finally, in our study of the real-space behavior of the correlation functions as a function of magnetization (field) and spin, we examine the functions $F_{R}(N)$ for higher spin, particularly $S=1$. Figure 9 compares extrapolated values for the nearest-neighbor correlation, $F_{1}(\infty)$, for spin 1 and spin $\frac{1}{2}$. For $S=\frac{1}{2}$ the curve is obtained by fitting a smooth curve through points for $F_{1}(14), F_{1}(12)$, and $F_{1}(10)$, as indicated. For $S=1$, points for $F_{1}(12)$ and $F_{1}(10)$ are sufficient. The curves have a quite different form. Change of curvature and an inflection point are apparent for $S=\frac{1}{2}$ but not for $S=1$. However, both curves do appear to start off from saturation in a similar manner. Corresponding curves for spin 1 and spin $\frac{1}{2}$ for the

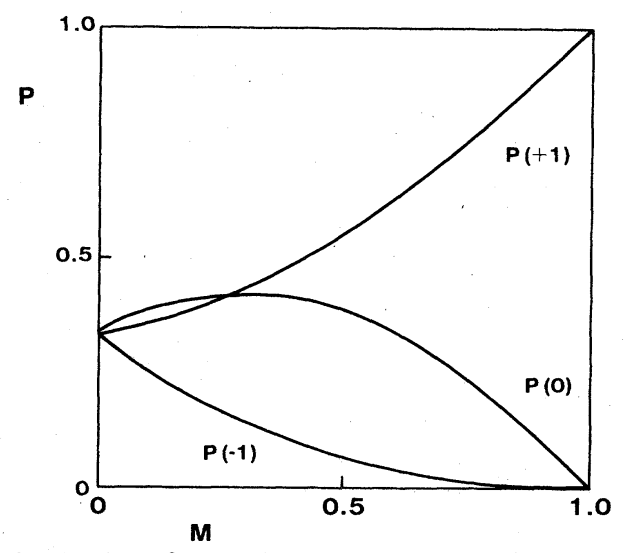

FIG. 8. A plot of the relative probability of a spin's being in the three allowed quantum states $S^{z}=+1,0$, and -1 for spin 1 , as a function of magnetization.

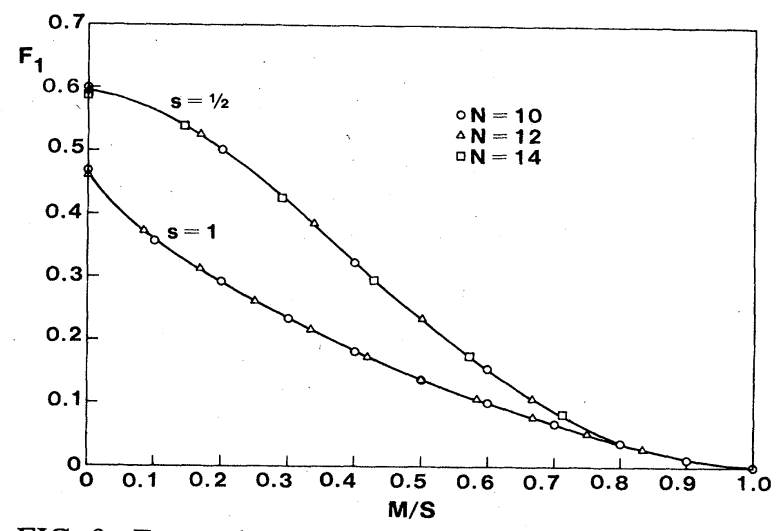

FIG. 9. Extrapolated values for the nearest-neighbor correlation function, $F_{1}(\infty)$, for spin 1 and spin $\frac{1}{2}$.

second-neighbor correlation function $F_{2}$ appear in Fig. 10. Both curves start out positive as $M$ increases from zero, pass through zero to negative values, and then increase back to zero as $M / S \rightarrow 1$. It is striking that the fluctuations are so much reduced in relative magnitude in the case of spin 1. What is perhaps more striking, however, is the fact that the pronounced oscillations about the zero value which occur for spin $\frac{1}{2}$ for $R>2$ are not apparent in the corresponding curves for spin 1. (Some oscillatory behavior occurs near saturation but the correlations do not change sign.) The virtual disappearance of oscillations directly implies that the tracking soft mode which is such a prominent feature for spin- $\frac{1}{2}$ chains is considerably reduced in importance for spin 1. This is true also for curves for $S>1$, and fluctuations are even less apparent for $S>1$ than for $S=1$. Our observations on the real-space correlation functions lead us to conclude that curves for $S \geq 1$ are strikingly different from those for spin $\frac{1}{2}$, and are much more classical in nature.

Our deductions concerning the relative unimportance of a soft mode for $S \geq 1$ are strengthened by a study of the Fourier transform, $S^{\mathrm{zz}}(q)$, of the real-space correlations. A plot of $S^{z z}(q)$ for Heisenberg AFM chains as a function

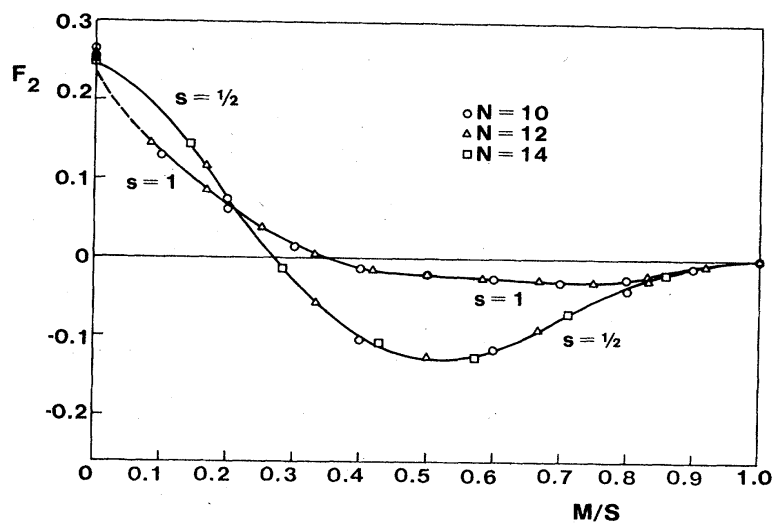

FIG. 10. Extrapolated values for the second-neighbor correlation function, $F_{2}(\infty)$, for spin 1 and spin $\frac{1}{2}$. Extrapolation is uncertain for spin 1 near $M=0$, as is indicated by the fact that the curve is shown dashed. 


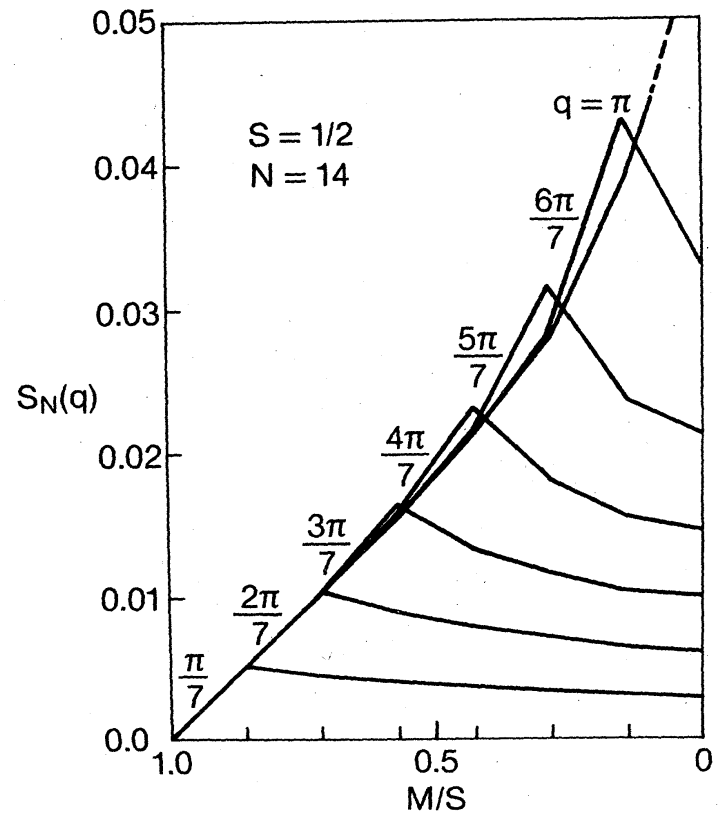

FIG. 11. Plot of the integrated intensity $S^{z z}(q)$ as a function of magnetization for various wave vectors $q$, for $N=14$ and $S=\frac{1}{2}$. The soft-mode feature is reflected in the pronounced cusps which occur for $q<\pi$.

of wave vector $q$ for various values of field $H$ appears as Fig. 11 of Ref. 11(b). For each value of field (or, equivalently, $M$ ) the curves show a cusp at the $q$ value corresponding to the soft mode. If the curves are plotted instead as a function of $M$ for various $q$ values, a very similar picture emerges. The cusps are, in fact, accentuated in this type of plot, and are illustrated in Fig. 11 for spin $\frac{1}{2}$ and $N=14$. Corresponding plots for spin 1 , $N=12$ and 10 look qualitatively very different, as illustrated in Fig. 12, where $S^{z z}(q)$ is plotted versus $M$ for various $q$ values, for $S=1$ and $N=12$. The difference between Figs. 11 and 12 is clearly apparent. Cusps can still be observed in the magnetization regime $1 \gtrsim M \gtrsim 0.5$, but they are evidently much less pronounced than in the case of spin $\frac{1}{2}$. In the region $0.5 \geq M>0$, no cusp structure appears. The cusp is a direct reflection of a tracking soft mode, as we shall observe in the next section, where we study dispersion spectra in detail, and a tracking soft mode, it turns out, is a quantum phenomenon, significant in the "quantum" magnetization region and barely apparent in the "classical" region. We have no explanation, at present, for the occurrence of maxima in the curves for all $q$ in the classical magnetization region. ${ }^{52}$

Appendix B comprises a set of tables (Tables VI-IX) of values of the correlation functions $F_{R}(N)$ corresponding to the longest chains for a given spin value. The correlation functions are derived from the eigenvectors corresponding to the lowest eigenvalues for a given $S_{T}^{z}$ (or magnetization $M$ ).

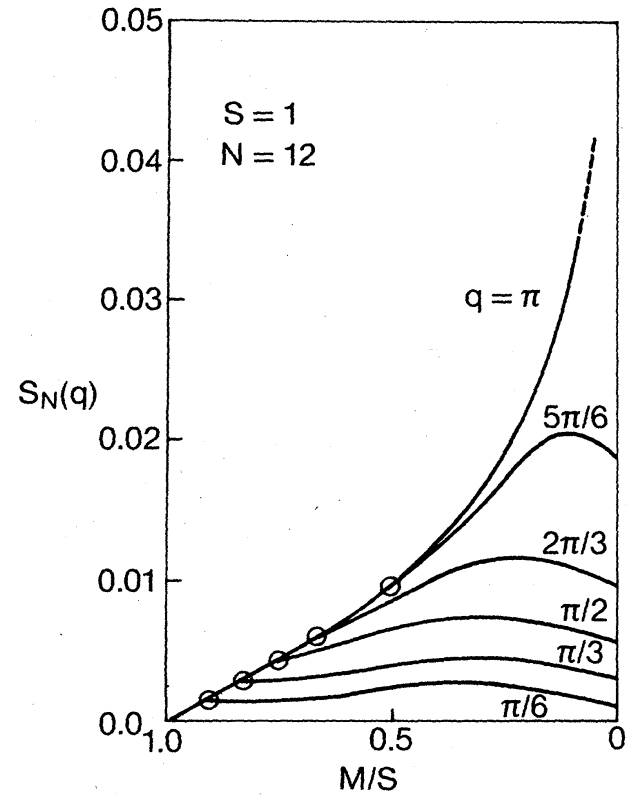

FIG. 12. Plot of the integrated intensity $S^{z z}(q)$ as a function of magnetization for various wave vectors $q$, for $N=12$ and $S=1$. (Correlation functions were not calculated for $N=14, S=1$, only the lowest-lying eigenvalues.) The softmode cusps are less pronounced in the case of spin 1, and occur only in the quantum regime, $1 \geqq M / S \geqq 0.5$.

\section{DISPERSION SPECTRA}

In discussing magnetization curves and correlation functions above, it has become apparent that spin chains with $S>\frac{1}{2}$ show significantly more classical character than chains with $S=\frac{1}{2}$. However, the manner and mechanism by which the classical features manifest themselves is far from apparent. A hint is provided by the $T=0$ magnetization isotherms which show quantum features only in the region close to saturation.

Dispersion spectra of excitations as a function of wave vector $q$ are a sensitive and informative property, and the low-lying excitations determine the properties of the system in question as $T \rightarrow 0$. Hence we have calculated the two lowest-lying eigenvalues as a function of $q$ for all values of $S_{T}^{z}$ for Heisenberg chains with $S=\frac{1}{2}, 1, \frac{3}{2}$, and 2. Interestingly, the phenomena we shall discuss are not related to the Haldane conjecture, which is irrelevant in the sense that all excitations are measured with respect to the lowest excitation for a given $S_{T}^{z}$. In other words, our discussions relate to a situation where the magnetization is always nonzero.

We are interested in the manner in which the fielddependent ( $S_{T}^{z}$-dependent) excitations for the extreme quantum limit, $S=\frac{1}{2}$, change as the spin increases. Specifically we are interested in the quantum-classical crossover mechanism. In Fig. 13 the classical dispersion curves $^{59}$ are shown in comparison with the curves for spin $\frac{1}{2}$. It was pointed out by Müller et al. ${ }^{11(b)}$ that the spin- 


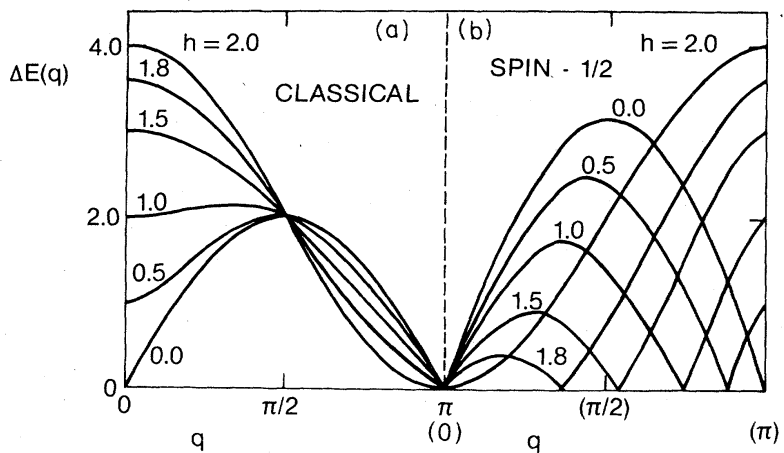

FIG. 13. Comparison of (a) classical dispersion curves as a function of reduced field $h$ in the thermodynamic limit with (b) quantum, spin- $\frac{1}{2}$, dispersion curves (lower boundaries of continua) for the same reduced fields $h$.

$\frac{1}{2}$ curves of Fig. 13 are actually the lower boundaries of spin-wave double continua (SWDC), whereas the classical curves are single-branch dispersion spectra. Restricted by the Lanczös technique to finding only the two lowest eigenvalues for a given $q$, we have not been able in this study to look for SWDC, which are such a striking feature of $S=\frac{1}{2}$ chains, in chains of higher spin. Aside from the question of SWDC, it is clear from Fig. 13 that the classical and quantum curves are very different in another respect. The classical curves have a soft mode at the zone boundary for all field values up to and including the saturation field, but a gap appears at the zone center for $H>0$, which is proportional to field. The spin- $\frac{1}{2}$ dispersion curves are those relevant for longitudinal dynamical correlation functions (the dispersion curves for transverse dynamical correlation functions are equivalent to those of Fig. 13 but are reflected about $q=\pi / 2$. They, in fact, correspond to the classical curves which are also relevant to transverse correlations). The dominant aspect of the spin- $\frac{1}{2}$ curves is that, in addition to a soft mode (zero-frequency excitation) at the zone center which persists for all $H$, there is a second soft mode which "tracks" across the Brillouin zone from edge to center as the field increases. ${ }^{50,51,11(\mathrm{~b})}$ The distance from $q=\pi$ is given by $\Delta q=2 \pi M$, i.e., the distance tracked by the soft mode is proportional to the magnetization. ${ }^{11}$ For $H>0$, a gap appears at $q=\pi$, which is proportional to field. (In this respect the $S=\frac{1}{2}$ and classical curves do resemble each other.)

Figure 14 shows the corresponding spin- $\frac{1}{2}$ dispersion curves for finite $N$, namely $N=14$. The curves are defined by points at the allowed $q$ values, $q=2 \pi / N$, $r=0,1,2, \ldots, N / 2$, joined by straight lines. Each curve is labeled by the value of $S_{T}^{z}$, where $M=N^{-1} S_{T}^{z}$. For finite $N$ the tracking mode is not soft since it has an excitation energy $O\left(N^{-1}\right)$ going to zero in the $N \rightarrow \infty$ limit. The excitation energies of these tracking modes appear to lie on a smooth curve (shown dashed) which varies according to the value of $N$. The curves overall show a resemblance to the limiting curves of Fig. 13, except in one important respect. Observe the curve for $S_{T}^{z}=5$. In

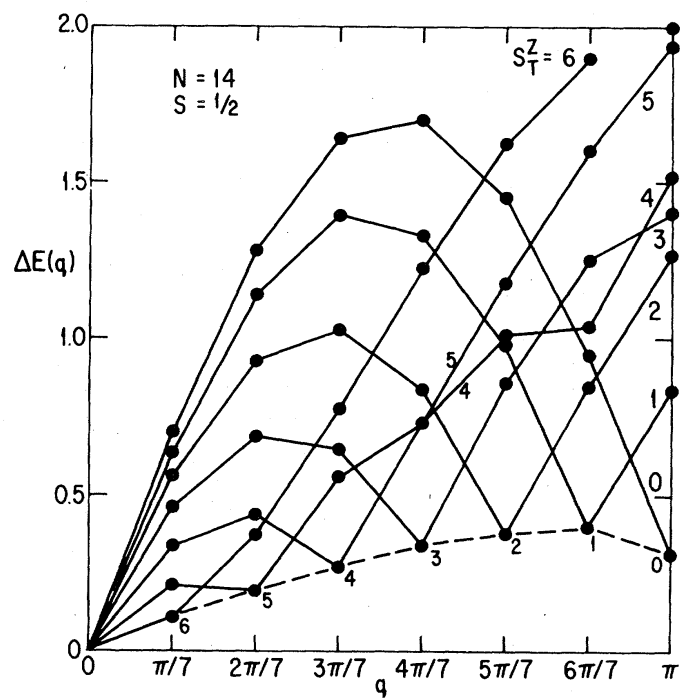

FIG. 14. Spin- $\frac{1}{2}$ dispersion curves for $N=14$ as a function of $S_{T}^{z}$ (magnetization). The soft-mode excitation features are apparent and extrapolate to zero as $1 / N$ in the thermodynamic limit.

addition to the "quasisoft" mode at $q_{1}=2 \pi / 7$, a second "quasisoft" mode ${ }^{52}$ is apparent at $q_{2}=2 q_{1}=4 \pi / 7$. Furthermore, in the case of the curve for $S_{T}^{z}=3$, a second soft mode appears as a depressed excitation energy at $q=\pi$ in addition to the first mode at $q=4 \pi / 7$. There is evidence even for small finite $N$ of a periodicity in $q$ in the thermodynamic limit which increases with increasing field. For finite- $N$ calculations the periodicity is always commensurate, but the periodicity may be commensurate or incommensurate in the thermodynamic limit. This phenomenon, which is characteristic of a Heisenberg chain, is discussed in Ref. 11(b). It has been extensively studied by Aghahosseini and Parkinson ${ }^{52}$ using both finite-chain and Bethe-ansatz calculations. The presence of periodicity in $q$ space has already been noted in Sec. III in relation to the behavior of the spin- $\frac{1}{2}$ real-space correlation functions in a field, and their Fourier transforms.

However, it is clear from the discussion above, that instead of a single field-dependent soft mode as calculated in Refs. 51 and 11(b), a multiplicity of soft modes, depending on the periodicity and hence on the field, will appear in long chains. ${ }^{52} \mathrm{~A}$ resolution of this inconsistency can be made by distinguishing between "static" and "dynamic" excitations. By static excitations we mean to imply excitations which contribute to the static properties, whereas dynamic excitations are a subset of the static excitations which contribute to the dynamical correlation function at $T=0$. This work is concerned with static properties, whereas the curves of Fig. 13 relate to dynamic excitations, i.e., excitations which are experimentally measurable by neutron scattering techniques. It is known that selection rules exist ${ }^{11}$ for the $T=0$ dynamics which restrict the classes of excitations which contribute. These selection rules concern restrictions on the total spin $S_{T}$ and its component $S_{T}^{z}$ that determine which excited states 
have spectral weight, i.e., have nonzero matrix elements with the ground state. One might therefore suspect that selection rules which forbid the contribution of large classes of static excitations might explain the discrepancy. It is very interesting that this is not so. Furthermore, the work of Aghahosseini and Parkinson ${ }^{52}$ (see also Ref. 51) makes it clear that static rather than dynamic excitations are the lowest-lying excitations over a fraction of the Brillouin zone (depending on field).

These particular "static" excitations have the same $S_{T}$ and $S_{T}^{z}$ as the dynamic excitations, and hence their contribution to the spectral weight is not forbidden by selection rules. In fact, it may be observed [see Figs. 15(c) and 18(d) of Ref. 11] that these special static states do carry some spectral weight, but it is very small in comparison with that carried by the Ishimura-Shiba dynamic excitations. Whether any weight persists in the thermodynamic limit from these special states has not been determined. In fact, such a determination would involve spindynamics calculations on much longer chains than can be handled at present. However, the point is made that classes of states which dominate the dynamics, i.e., are experimentally observable in real Heisenberg system, may differ from those classes of states which determine the low-temperature static properties.

Now, in Fig. 15 we turn to the dispersion spectra for a spin-1 chain of $N=14$ spins. Apart from a factor of 2 in the energy scale (suggesting an appropriate normalization of $\Delta E / S$ for the excitations) the qualitative agreement with the plot for spin $\frac{1}{2}$ is extremely striking. Again a soft mode is observed which duly tracks across the Brillouin zone. It is relatively less pronounced than in the case of spin $\frac{1}{2}$, particularly for $\pi / 2 \leqslant q<\pi$. Additionally, the curves for $S_{T}^{z}=12$ and 11 show secondary soft modes, i.e., evidence of magnetization-dependent periodicity in $q$ in the thermodynamic limit, in direct analogy with the situation for spin $\frac{1}{2}$. The major point of difference is that Fig. 15 comprises only curves for $N S-1 \geqq S_{T}^{z} \geqq N S$ $-N / 2$. Curves for $N S-N / 2>S_{T}^{z} \geqq 1$ do not appear. Plotted on a separate figure (not shown), the curves for $N S-N / 2 \geqq S_{T}^{z}>1$ yield a confusing picture, not easily interpreted, but very different from Figs. 13 and 14. The behavior of the residual curves will be discussed subsequently in detail since it is the focal point of this paper. Figures clearly equivalent to Figs. 14 and 15 are obtained for spin chains with $S=\frac{3}{2}$ and 2 . The moving (primary) soft mode and incipient periodicity are clearly observable for all values of spin. The only difference is the fact that the primary soft mode becomes progressively less pronounced as the spin value increases. For all spin values the soft mode points appear to lie on a smooth curve, shown dashed in Figs. 14 and 15. For all $S>\frac{1}{2}$ a softmode point exists at $q=\pi$, and in all cases a $1 / N$ extrapolation of this point proceeds smoothly to zero in the $N \rightarrow \infty$ limit. This implies that the primary soft-mode points for all $q$ also go to zero as $N \rightarrow \infty$ (thus justifying the term soft mode)! This feature is known rigorously to be the case for spin $\frac{1}{2}$, of course. Hence it seems clearly established that a fraction of the dispersion curves for all $S$ possess the salient features of the entire set of curves for

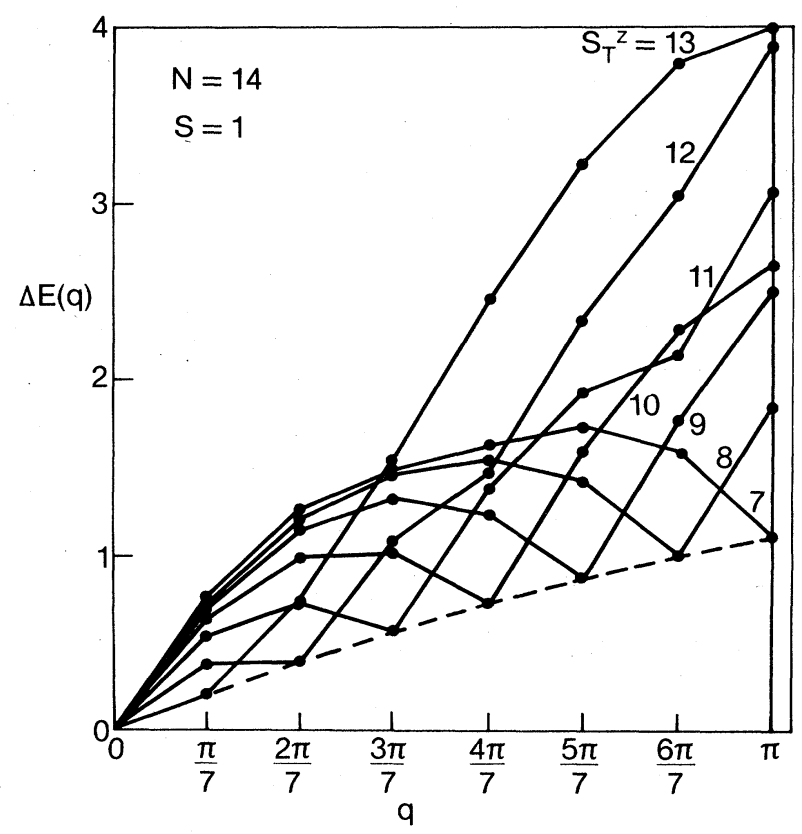

FIG. 15. Spin-1 dispersion curves for $N=14$ as a function of $S_{T}^{z}$. The qualitative resemblance to the corresponding curves of Fig. 14 is apparent. Again, the soft-mode features extrapolate to zero in the thermodynamic limit.

spin $\frac{1}{2}$. Note that the spectra which have the spin- $\frac{1}{2}$ (henceforth called quantum) character involve those states which define the quantum region of the magnetization curves, namely $M_{s}>M>M_{s}(1-1 / 2 S)$. Hence the information gathered from our various investigations is consistent.

Although our study is concerned with antiferromagnets, it is of interest to note that existing calculations of Jain et al. ${ }^{60}$ on ferromagnetic chains of higher spin are consistent with our interpretations in indicating that a fraction of the dispersion spectra have the characteristics of those for spin- $\frac{1}{2}$ chains. In the case of spin- $\frac{1}{2}$ finite chains, bound states are well known ${ }^{32}$ to lie below the one-spin-wave single-branch dispersion curve and the two-spin-wave continuum. The bound-spin complexes are states with $S_{T}^{z}=N / 2-2, N / 2-3, \ldots, 1,0$ ( $N$ even), for a total of $(N / 2-1)$ distinct branches. For chains of higher spin similar bound-spin complexes appear, but only for $\quad S_{T}^{z}=N S-2, N S-3, \ldots,(N S-N / 2+1),(N S$ $-N / 2)$, always giving a total of $(N / 2-1)$ distinct branches. For $S>\frac{1}{2}$ no bound complexes with $S_{T}^{z}<N S-N / 2$ appear. In fact, such states are not apparent ${ }^{60}$ in the low-lying excitations of either bound or spin-wave character. The behavior of states with $S_{T}^{z}<N S-N / 2$ has not been studied and would be interesting. Again the states discussed include the states which define the quantum region of the magnetization curves.

Let us now consider the antiferromagnetic spin-1 dispersion curves having $S_{T}^{z}<N S-N / 2$ (states with $S_{T}^{z}=0$ are excluded since such states are anomalous according to the Haldane conjecture, and in any case we are 
considering spectral behavior corresponding to nonzero magnetization). An initial examination of the lowestlying states for a given $q$ gives a definite hint that the curves are displaying a qualitative resemblance to the classical curves of Fig. 13. However, there are many anomalous features. On closer examination it turns out that the soft-mode features so prominent in Fig. 15 persist also for low $S_{T}^{z}$ values (low field), but are only significant for $q$ values near $\pi$. They appear to persist over what amounts to a second Brillouin zone, but this has to be verified from an examination of higher excitations, not just lowest-lying excitations only. It appears in this second regime that both quantum (soft mode) and classical features are present simultaneously. Which excitations dominate, i.e., are experimentally observable, could in principle be determined by a calculation of the $T=0$ dynamics. Such a calculation is barely feasible for chains of length and spin value considered here, however. Hence, having noted that the soft-mode features in the quantum zone become less prominent with increasing spin, we examine dispersion curves for $S_{T}^{z}<N S-N / 2$ for $S=\frac{3}{2}$ and $N=10$ in Fig. 16. The equivalent curves for $S=\frac{3}{2}$ and $N=8$ are essentially similar and are not shown. For spin $\frac{3}{2}$ softmode effects are much less apparent than for spin 1 in this "classical" regime, and appear for $N=10$ only for $q \geqq 4 \pi / 5$. Allowing for these factors, the qualitative resemblance of the dispersion curves of Fig. 16 to the classical curves of Fig. 13 is extremely striking and suggestive. As $S_{T}^{z}$ (field) increases, the curves, all of which start out at zero frequency, start to cross, and the curves of higher $S_{T}^{z}$ head upward, indicating a field-dependent energy gap at $q=\pi$. For low $q, q=\pi / 5$ and $2 \pi / 5$, the curves decrease monotonically with increasing $S_{T}^{z}($ field), in agreement with the classical picture. At $q=\pi / 2$ the classical amplitude is given by $\Delta E / S=2.0$. For $S=\frac{3}{2}$ the curves are starting to cross in the vicinity of $\pi / 2$, and the amplitude, though not precisely defined due to finitesize effects, is in reasonable agreement with the classical expectation. These features are also apparent in corresponding curves for $S=\frac{3}{2}, N=8$ and $S=2, N=8$. In the case of $S=\frac{3}{2}$ and $N=10$ (Fig. 16), the curves for $q \geqq 4 \pi / 5$ now increase monotonically with increasing $S_{T}^{z}$ (field) up to $S_{T}^{z}=8$. The curves for the largest $S_{T}^{z}$ values of 9 and 10 are anomalously low, reflecting a persistence of the soft-mode phenomenon. Hence the finitechain dispersion curves for values of $S_{T}^{z}<N S-N / 2$ for $S=\frac{3}{2}$ and 2 clearly display classical tendencies, with slight residual anomalies attributable to finite-size and quantum effects. The borderline case, $S=1$, needs special discussion. A Lanczös technique which retains the two lowest states enables us to discard those states which have a clear soft-mode character. These are the lowest states with $q=q_{0}$, where $q_{0}=2 \pi S_{T}^{z} / N$ for $1 \leqq S_{T}^{z} \leqq N / 2-1$. In plotting the dispersion curves shown in Fig. 17 these states were replaced by the next-lowest state, indicated by a circled point. It is possible that adjacent points for a given $S_{T}^{z}$ may in some cases also belong to the soft-mode branch, although considerably longer chains would be necessary to establish this clearly. Bearing in mind this uncertainty, the curves shown now have the same general

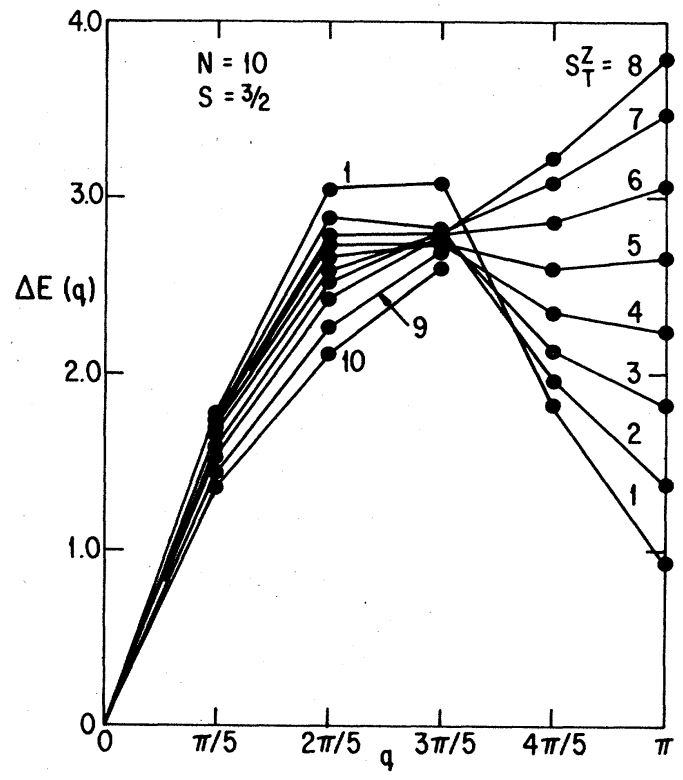

FIG. 16. Dispersion curves for spin $\frac{3}{2}, N=10$ in the nonquantum regime. These curves are very different from the quantum dispersion curves of Figs. 14 and 15, and show a strong qualitative resemblance to the classical dispersion curves of Fig. 13(a).

character as the corresponding ones for $S=\frac{3}{2}$ and $S=2$. They also appear to show reasonable agreement with the main features of the "classical" dispersion curves [Fig. 13(a)].

Our finite-chain calculations for higher spin at nonzero field appear to establish a remarkable quantum-classical crossover effect. Dispersion curves for $S_{T}^{z}$ values near the antiferromagnetic saturation limit, numbering $(N / 2-1)$ curves in all, show quantum character, including periodicity and soft modes in the thermodynamic limit, which are analogous to the entire set of dispersion curves for $S=\frac{1}{2}$. Whether spin-wave double continua (SWDC) are present also cannot be determined since Lanczös techniques only find a restricted number of eigenvalues. It seems likely that SWDC do exist, and their contribution to the $T=0$ dynamics in the field region below the saturation field would be interesting to determine. Dispersion curves for all other values of $S_{T}^{z}$ display a completely different character, approximating quite closely the classical single-branch dispersion curves. Whether these finite$S$ curves are analogous to the classical curves whose $T=0$ spectral weight manifests itself as a delta function located along the branch is another interesting question. It is clear, however, that the classical-type excitations dominate the static properties, $T=0$ magnetization, and correlation functions, in the appropriate field (or magnetization) regime. The situation is very simple, though unexpected, as is shown by the sketch, Fig. 18, which is a plot of spin value versus magnetization showing the crossover between the two major regimes, classical and quantum. 


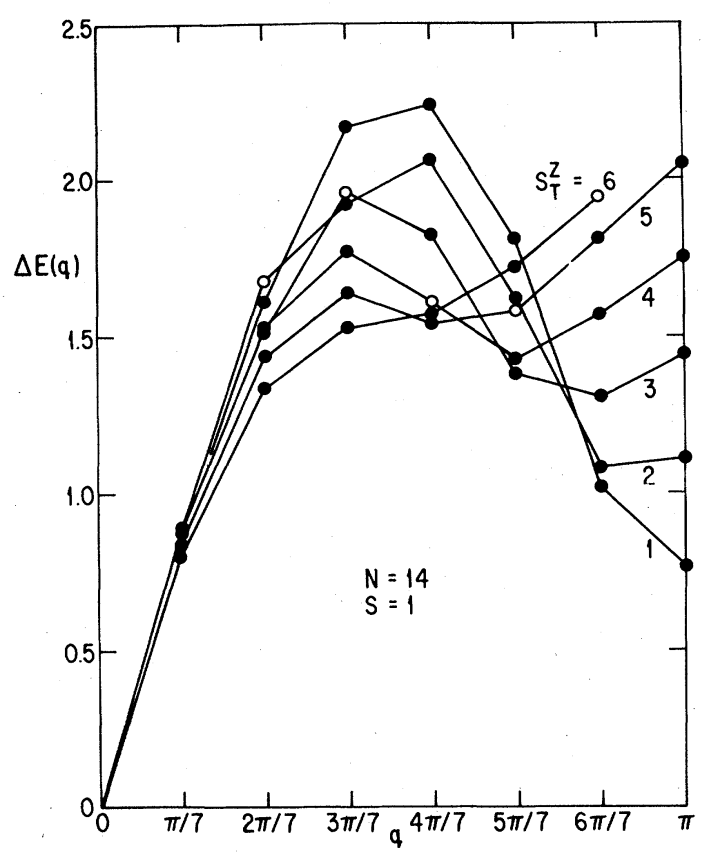

FIG. 17. Dispersion curves for spin $1, N=14$, for $S_{T}^{z}$ values $<N / 2$. Lower-lying excitations corresponding to coexisting soft-mode features have been omitted where appropriate, and the next-highest excitation denoted by an open circle shown instead. A strong qualitative resemblance is apparent to the classical curves of Fig. 13(a) and the curves of Fig. 16.

Figure 18 illustrates the fact that the quantum behavior characteristic of spin $\frac{1}{2}$ persists for all $S$ over a magnetization regime of magnitude $M_{s} / 2 S$, thus losing significance as $S \rightarrow \infty$.

From the dispersion spectra it is clear that the crossover is not completely sharp. An intermediate crossover regime exists, as indicated in Fig. 18, where soft-mode features can still be distinguished. In the case of spin 1, therefore, quantum dispersion features coexist with classical dispersion features in the entire nonquantum regime. The impact of these quantum features is minimal as far as magnetization curves and correlation functions are concerned. The impact on dynamic properties is not at present known.

The eigenvalue tables of Appendix A contain representative data as a function of wave vector $q$ on which our analysis of dispersion spectra in this section has been based.

\section{SUMMARY AND DISCUSSION}

Our finite-chain studies of $S=\frac{1}{2}$ and $S>\frac{1}{2}$ Heisenberg antiferromagnets have revealed a most unusual crossover phenomenon. The characteristic dispersion spectra of $S=\frac{1}{2}$ chains, where a soft mode tracks across the Brillouin zone as the field varies, appear also in chains with higher spin. However, for $S>\frac{1}{2}$, the field (or magnetization) region involved is only a fraction of the total regime,

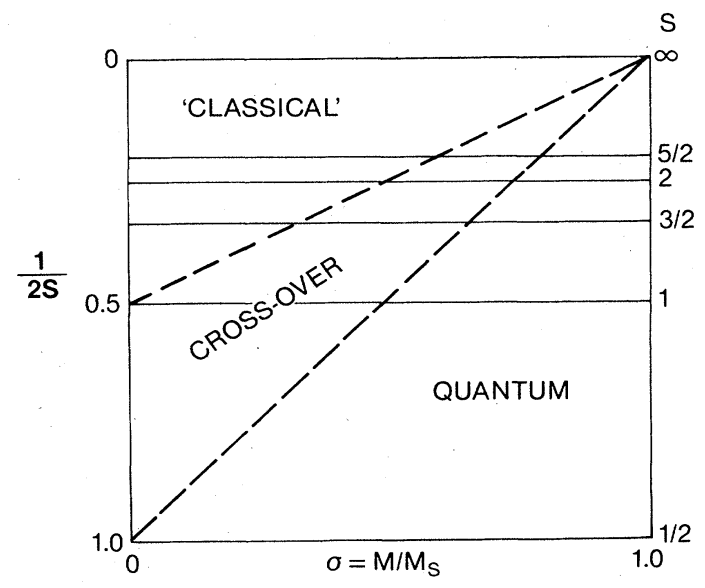

FIG. 18. A plot of $1 / 2 S$ versus reduced magnetization which summarizes conclusions concerning quantum-classical crossover. Two major regions, quantum and classical are evident, together with an intermediate crossover region, as discussed in the text.

$M / 2 S$ in extent, close to the saturation limit. The remaining dispersion curves behave quite differently, displaying a marked classical character. Hence spin chains show the quantum-classical crossover behavior illustrated in Fig. 18. The relative extent of the quantum region shrinks as the spin value increases, disappearing in the $S \rightarrow \infty$ limit. The crossover from quantum to classical behavior is not sharp. An intermediate regime exists where quantum soft-mode features coexist with low-lying excitations of "classical" type. For $S>1$ a third region is indicated where only classical-type excitations are apparent. No soft-mode features are observable in the lowest-lying excitations.

It is important to note, however, that our identification of quantum, intermediate and "classical" regimes is based solely on the behavior of the two lowest-lying excitations for a given $S_{T}^{z}$ and $q$. The presence of tracking soft modes in a field is only one characteristic of quantum behavior. The other is the presence of extended spin-wave double continua (SWDC) which have a continuous distribution of spectral weight in energy across the continuum at a given value of $q$. An existing theory ${ }^{27,61}$ of quantum-classical crossover in spin-dynamical behavior (in zero field) postulates that SWDC persist for all $S$, but that the spectral weight becomes increasingly concentrated in the vicinity of the lower boundary of the SWDC as $S$ increases. Our use of the Lanczös approach precludes a search for SWDC. An investigation of dynamic properties would be extremely interesting, but is currently a challenging project in view of the size of systems involved. The striking resemblance of the quantum region for $S>\frac{1}{2}$ to the well-studied region for $S=\frac{1}{2}$, together with indirect supporting evidence from the static correlation functions (or integrated intensity), suggests the dynamical behavior in the quantum regime for $S>\frac{1}{2}$ will parallel that for $S=\frac{1}{2}$. We are not in a position to make a conjecture about the dynamical behavior of the "classi- 
cal" regime, however. The excitation branches identified here may be true single-branch dispersion spectra of classical type or they may be lower boundaries of a new class of SWDC, different from the SWDC of the quantum regime. If the latter turns out to be the case, and if there is a distribution of spectral weight above the lower boundary, we should conclude some residual degree of quantum character exists even in the "classical" regime for $S<\infty$. Clearly, further investigation of such interesting possibilities should be pursued.

The behavior of the static properties reflects the quantum (high-field) to "classical" (low-field) crossover. The $T=0$ magnetization isotherms for $S>1$ show a marked linear character in the classical regime, switching over to quadratic behavior near the saturation field in the quantum regime. The Fourier-transformed spin-spin correlations as a function of wave vector $q$ or magnetization $M$ show a pronounced cusp corresponding to the tracking soft mode for spin $\frac{1}{2}$. This cusp results from a periodicity in $q$ space which reflects a pronounced oscillatory character in the spin- $\frac{1}{2}$ real-space correlations. The situation for $S>1$ is dramatically different. The cusp effects in $S(q)$ are still discernible in the quantum regime but are no longer prominent. The oscillatory character of the corresponding real-space correlations almost disappears, slight residual effects persisting in the quantum regime, as would be expected. Fluctuation effects in the correlations for $S>1$ are markedly reduced.

One motivating factor for this study has been a conjecture by Haldane ${ }^{2}$ which predicts that the class of integerspin Heisenberg AFM chains will have a gap in the spectral excitations between the ground-state singlet and higher excitations, whereas the class of half-integer-spin Heisenberg chains has a gapless excitation spectrum. We have extended previous numerical work of Kolb, Botet, and Jullien, ${ }^{6}$ and others, ${ }^{8-10}$ in particular by examining, effectively, excitations in a nonzero magnetic field. None of our additional studies is inconsistent with the Haldane conjecture. However, we do note that convergence problems appear to be more severe for $S>\frac{1}{2}$ than for $S=\frac{1}{2}$, and that our data could also be interpreted as supporting the alternative hypothesis that Heisenberg chains with $S>\frac{1}{2}$ behave differently from chains with $S=\frac{1}{2}$. This would be in accordance with our conclusions above concerning quantum-classical crossover.

Such are our major emphases and conclusions. We might note two other features of interest:

(a) A preliminary study of the long-range order for $S>\frac{1}{2}$ has turned out to be much less revealing than a previous study for $S=\frac{1}{2}, 32$ apparently illustrating the enhanced convergence problems noted above.

(b) The magnetization and autocorrelation function of spin-1 Heisenberg chains have provided insight into the behavior of spin deviations as a function of field. Near saturation, the relevant excitations correspond to single deviations at a site, analogous to the case of spin $\frac{1}{2}$. The probability of having two deviations at a site is negligible in the quantum (high-field) region, but becomes appreciable in the low-field region.

\section{ACKNOWLEDGMENTS}

We are happy to acknowledge M. F. Thorpe for fruitful discussions and useful suggestions and G. Müller for several useful comments and a critical reading of the manuscript. This work was supported by U. S. National Science Foundation (NSF) Grant No. DMR-77-09170 and the U. S. Office of Naval Research (ONR) and, in part, by NSF Grant No. DMR-80-10819 and the North Atlantic Treaty Organization (NATO).

\section{APPENDIX A}

Tables I-V of the lowest two energy levels for each $S_{T}^{z}, q$ for various $N, S$. In each case the energy is measured relative to the aligned state (ferromagnetic ground state) which is the highest state for the antiferromagnet. Parentheses indicate that the state is a lower $S_{T}^{z}$ component of a state with higher $S_{T}$. A state which does not exist is indicated by a dash.

TABLE I. Table of the lowest two energy levels for each $S_{T}^{z}, q$ for $N=14, S=1$.

\begin{tabular}{|c|c|c|c|c|c|c|c|c|}
\hline$S_{T}^{2} q$ & 0 & $\pi / 7$ & $2 \pi / 7$ & $3 \pi / 7$ & $4 \pi / 7$ & $5 \pi / 7$ & $6 \pi / 7$ & $\pi$ \\
\hline 14 & $0^{-}$ & - & - & - & - & - & - & - \\
\hline 13 & (0) & $\overline{0.1981}$ & $\overline{0.7530}$ & $\overline{1.5550}$ & $\overline{2.4450}$ & $3 . \overline{2470}$ & $3 . \overline{8019}$ & $4^{-}$ \\
\hline 12 & $\begin{array}{l}7.1594 \\
7.9001\end{array}$ & $\begin{array}{l}6.5308 \\
7.5180\end{array}$ & $\begin{array}{l}6.8346 \\
7.5125\end{array}$ & $\begin{array}{l}5.9924 \\
6.8110\end{array}$ & $\begin{array}{l}5.9302 \\
6.4273\end{array}$ & $\begin{array}{l}5.0407 \\
5.5444\end{array}$ & $\begin{array}{l}4.6584 \\
4.8631\end{array}$ & $\begin{array}{c}4 \\
(4)\end{array}$ \\
\hline 11 & $\begin{array}{l}8.5424 \\
8.5434\end{array}$ & $\begin{array}{l}8.7792 \\
9.4716\end{array}$ & $\begin{array}{l}9.3162 \\
9.6860\end{array}$ & $\begin{array}{r}9.5867 \\
10.2321\end{array}$ & $\begin{array}{l}10.1110 \\
11.0463\end{array}$ & $\begin{array}{l}10.3189 \\
10.8811\end{array}$ & $\begin{array}{l}10.4197 \\
11.0759\end{array}$ & $\begin{array}{l}10.5917 \\
11.6098\end{array}$ \\
\hline 10 & $\begin{array}{l}13.8550 \\
15.0632\end{array}$ & $\begin{array}{l}13.5566 \\
14.4279 \\
\end{array}$ & $\begin{array}{l}13.6566 \\
14.0751\end{array}$ & $\begin{array}{l}13.3036 \\
14.0502 \\
\end{array}$ & $\begin{array}{l}13.2337 \\
14.3387\end{array}$ & $\begin{array}{l}12.6127 \\
13.4587\end{array}$ & $\begin{array}{l}12.5636 \\
12.7873\end{array}$ & $\begin{array}{l}12.4040 \\
12.4041\end{array}$ \\
\hline
\end{tabular}


TABLE I. (Continued).

\begin{tabular}{|c|c|c|c|c|c|c|c|c|}
\hline$S_{T}^{2}$ & 0 & $\pi / 7$ & $2 \pi / 7$ & $3 \pi / 7$ & $4 \pi / 7$ & $5 \pi / 7$ & $6 \pi / 7$ & $\pi$ \\
\hline 9 & $\begin{array}{l}15.6863 \\
15.7311\end{array}$ & $\begin{array}{l}15.4832 \\
16.4656\end{array}$ & $\begin{array}{l}16.1486 \\
17.3610\end{array}$ & $\begin{array}{l}16.1599 \\
16.9990\end{array}$ & $\begin{array}{l}16.4901 \\
16.9084\end{array}$ & $\begin{array}{l}16.7793 \\
17.0913\end{array}$ & $\begin{array}{l}16.5240 \\
17.5437\end{array}$ & $\begin{array}{l}16.8909 \\
18.2319\end{array}$ \\
\hline 8 & $\begin{array}{l}19.6686 \\
21.1177\end{array}$ & $\begin{array}{l}19.2514 \\
20.3967\end{array}$ & $\begin{array}{l}19.6229 \\
19.9078\end{array}$ & $\begin{array}{l}19.2196 \\
19.6541\end{array}$ & $\begin{array}{l}19.1520 \\
19.5672\end{array}$ & $\begin{array}{l}18.8489 \\
19.6990\end{array}$ & $\begin{array}{l}18.8180 \\
20.1168\end{array}$ & $\begin{array}{l}19.2138 \\
19.2602\end{array}$ \\
\hline 7 & $\begin{array}{l}22.6139 \\
22.6224\end{array}$ & $\begin{array}{l}21.7702 \\
22.1325\end{array}$ & $\begin{array}{l}21.5430 \\
21.9919\end{array}$ & $\begin{array}{l}21.5727 \\
22.1044\end{array}$ & $\begin{array}{l}21.7307 \\
22.2363\end{array}$ & $\begin{array}{l}22.1749 \\
22.4655\end{array}$ & $\begin{array}{l}21.6946 \\
22.9714\end{array}$ & $\begin{array}{l}22.1905 \\
23.7284\end{array}$ \\
\hline 6 & $\begin{array}{l}24.4473 \\
26.0641\end{array}$ & $\begin{array}{l}23.8765 \\
25.2648\end{array}$ & $\begin{array}{l}24.4475 \\
24.7232\end{array}$ & $\begin{array}{l}23.9708 \\
24.5358\end{array}$ & $\begin{array}{l}23.8086 \\
24.4930\end{array}$ & $\begin{array}{l}24.0556 \\
24.3421\end{array}$ & $\begin{array}{l}24.1200 \\
24.8688\end{array}$ & $\begin{array}{l}24.2839 \\
24.3189\end{array}$ \\
\hline 5 & $\begin{array}{c}25.9377 \\
(26.0641)\end{array}$ & $\begin{array}{l}26.1917 \\
26.3035\end{array}$ & $\begin{array}{l}26.5366 \\
26.8639\end{array}$ & $\begin{array}{l}26.1186 \\
26.5792\end{array}$ & $\begin{array}{l}25.9984 \\
26.4797\end{array}$ & $\begin{array}{l}26.4500 \\
26.6788\end{array}$ & $\begin{array}{l}25.8085 \\
27.2744\end{array}$ & $\begin{array}{l}26.4257 \\
28.1157\end{array}$ \\
\hline 4 & $\begin{array}{l}28.1171 \\
29.8693\end{array}$ & $\begin{array}{l}27.4646 \\
28.9939\end{array}$ & $\begin{array}{l}28.1932 \\
28.3440\end{array}$ & $\begin{array}{l}27.9494 \\
28.0958\end{array}$ & $\begin{array}{l}28.2553 \\
28.5926\end{array}$ & $\begin{array}{l}27.8777 \\
28.4392\end{array}$ & $\begin{array}{l}27.6965 \\
28.2949\end{array}$ & $\begin{array}{c}27.6271 \\
(28.1157)\end{array}$ \\
\hline 3 & $\begin{array}{c}29.2507 \\
(29.8693)\end{array}$ & $\begin{array}{l}29.1782 \\
30.0021\end{array}$ & $\begin{array}{l}29.2078 \\
29.9278\end{array}$ & $\begin{array}{l}29.2898 \\
29.4932\end{array}$ & $\begin{array}{l}29.3505 \\
30.0583\end{array}$ & $\begin{array}{l}29.6585 \\
29.7954\end{array}$ & $\begin{array}{l}28.9242 \\
30.4166\end{array}$ & $\begin{array}{l}29.5252 \\
31.3097\end{array}$ \\
\hline 2 & $\begin{array}{l}30.6801 \\
32.4227\end{array}$ & $\begin{array}{c}(30.0021) \\
31.5477\end{array}$ & $\begin{array}{l}30.7477 \\
31.3008\end{array}$ & $\begin{array}{l}30.2472 \\
30.4962\end{array}$ & $\begin{array}{c}(30.0583) \\
30.3621\end{array}$ & $\begin{array}{l}30.5251 \\
30.8984\end{array}$ & $\begin{array}{c}(30.4166) \\
31.3411\end{array}$ & $\begin{array}{c}30.6367 \\
(31.3097)\end{array}$ \\
\hline 1 & $\begin{array}{c}31.8149 \\
(32.4227)\end{array}$ & $\begin{array}{c}(31.5477) \\
32.1791\end{array}$ & $\begin{array}{c}(31.3008) \\
31.3907\end{array}$ & $\begin{array}{c}(30.4962) \\
30.9591\end{array}$ & $\begin{array}{c}(30.3621) \\
31.0304\end{array}$ & $\begin{array}{c}(30.8984) \\
31.5829\end{array}$ & $\begin{array}{c}(31.3411) \\
32.4532\end{array}$ & $\begin{array}{c}(31.3097) \\
33.1962\end{array}$ \\
\hline 0 & $\begin{array}{c}(32.4227) \\
33.6551\end{array}$ & $\begin{array}{l}(31.5477) \\
(32.1791)\end{array}$ & $\begin{array}{c}31.3381 \\
(31.3907)\end{array}$ & $\begin{array}{c}30.6156 \\
(30.9591)\end{array}$ & $\begin{array}{l}(30.3621) \\
(31.0304)\end{array}$ & $\begin{array}{l}(30.8984) \\
(31.5829)\end{array}$ & $\begin{array}{l}(31.3411) \\
(32.4532)\end{array}$ & $\begin{array}{c}31.6462 \\
(33.1962)\end{array}$ \\
\hline
\end{tabular}

TABLE II. Table of the lowest two energy levels for each $S_{T}^{z}, q$ for $N=12, S=1$.

\begin{tabular}{|c|c|c|c|c|c|c|c|}
\hline$S_{T}^{2} q$ & 0 & $\pi / 6$ & $\pi / 3$ & $\pi / 2$ & $2 \pi / 3$ & $5 \pi / 6$ & $\pi$ \\
\hline 12 & $0^{-}$ & - & - & - & - & - & - \\
\hline 11 & (0) & $0 . \overline{2679}$ & 1 & $2^{-}$ & $3-$ & $3 . \overline{7321}$ & $4^{-}$ \\
\hline 10 & $\begin{array}{l}6.8986 \\
7.8645\end{array}$ & $\begin{array}{l}6.1428 \\
7.3558\end{array}$ & $\begin{array}{l}6.4862 \\
7.3439\end{array}$ & $\begin{array}{l}5.5038 \\
6.4357\end{array}$ & $\begin{array}{l}5.3747 \\
5.9239\end{array}$ & $\begin{array}{l}4.4443 \\
4.8666\end{array}$ & $\begin{array}{c}3 \\
(4)\end{array}$ \\
\hline 9 & $\begin{array}{l}8.6895 \\
8.6899\end{array}$ & $\begin{array}{l}8.6563 \\
8.9898\end{array}$ & $\begin{array}{l}8.9280 \\
9.7016\end{array}$ & $\begin{array}{r}9.5929 \\
10.7265\end{array}$ & $\begin{array}{r}9.8790 \\
10.5285\end{array}$ & $\begin{array}{r}9.9881 \\
10.7893\end{array}$ & $\begin{array}{l}10.1990 \\
11.4768\end{array}$ \\
\hline 8 & $\begin{array}{l}13.2886 \\
14.7674\end{array}$ & $\begin{array}{l}12.9641 \\
13.9857\end{array}$ & $\begin{array}{l}13.1050 \\
13.5430\end{array}$ & $\begin{array}{l}12.6861 \\
13.4870\end{array}$ & $\begin{array}{l}12.5282 \\
13.8108\end{array}$ & $\begin{array}{l}11.7960 \\
12.7496\end{array}$ & $\begin{array}{l}11.8938 \\
11.9193\end{array}$ \\
\hline 7 & $\begin{array}{l}15.5136 \\
15.5990\end{array}$ & $\begin{array}{l}15.2002 \\
16.5861\end{array}$ & $\begin{array}{l}15.4024 \\
16.1890\end{array}$ & $\begin{array}{l}15.6662 \\
16.1617\end{array}$ & $\begin{array}{l}16.0200 \\
16.3892\end{array}$ & $\begin{array}{l}15.7204 \\
16.8992\end{array}$ & $\begin{array}{l}16.0783 \\
17.7244\end{array}$ \\
\hline 6 & $\begin{array}{l}18.5781 \\
20.3592\end{array}$ & $\begin{array}{l}18.1338 \\
19.4885\end{array}$ & $\begin{array}{l}18.5799 \\
18.9763\end{array}$ & $\begin{array}{l}18.1632 \\
18.7641\end{array}$ & $\begin{array}{l}18.0752 \\
18.5859\end{array}$ & $\begin{array}{l}18.1401 \\
18.5841\end{array}$ & $\begin{array}{l}19.0624 \\
19.0795\end{array}$ \\
\hline 5 & $\begin{array}{l}20.6380 \\
20.6963\end{array}$ & $\begin{array}{l}20.6941 \\
21.2755\end{array}$ & $\begin{array}{l}20.4195 \\
20.9938\end{array}$ & $\begin{array}{l}20.3705 \\
21.0979\end{array}$ & $\begin{array}{l}20.8155 \\
21.1987\end{array}$ & $\begin{array}{l}20.1964 \\
21.7451\end{array}$ & $\begin{array}{l}20.7896 \\
22.6729\end{array}$ \\
\hline 4 & $\begin{array}{l}22.6785 \\
24.6524\end{array}$ & $\begin{array}{l}22.0251 \\
23.6691\end{array}$ & $\begin{array}{l}22.7517 \\
23.0479\end{array}$ & $\begin{array}{l}22.5154 \\
22.9949\end{array}$ & $\begin{array}{l}23.0970 \\
23.2036\end{array}$ & $\begin{array}{l}22.4608 \\
22.9190\end{array}$ & $\begin{array}{c}22.3089 \\
(22.6729)\end{array}$ \\
\hline 3 & $\begin{array}{c}24.0121 \\
(24.6524)\end{array}$ & $\begin{array}{l}23.9152 \\
24.8327\end{array}$ & $\begin{array}{l}24.0799 \\
24.7825\end{array}$ & $\begin{array}{l}24.3908 \\
24.8176\end{array}$ & $\begin{array}{l}24.4261 \\
24.5807\end{array}$ & $\begin{array}{l}23.6951 \\
25.2560\end{array}$ & $\begin{array}{l}24.2343 \\
26.2781\end{array}$ \\
\hline 2 & $\begin{array}{l}25.4897 \\
27.5294\end{array}$ & $\begin{array}{c}(24.8327) \\
26.5061\end{array}$ & $\begin{array}{l}25.6695 \\
26.1920\end{array}$ & $\begin{array}{l}25.2078 \\
25.4703\end{array}$ & $\begin{array}{l}25.4379 \\
25.8410\end{array}$ & $\begin{array}{c}(25.2560) \\
26.3359\end{array}$ & $\begin{array}{c}25.5218 \\
(26.2781)\end{array}$ \\
\hline
\end{tabular}


TABLE II. (Continued).

\begin{tabular}{cccccccc}
\hline \hline$q$ & 0 & $\pi / 6$ & $\pi / 3$ & $\pi / 2$ & $2 \pi / 3$ & $5 \pi / 6$ \\
$S_{T}^{z}$ & & & & & & \\
\hline 1 & 26.8058 & $(26.5061)$ & $(26.1920)$ & $(25.4703)$ & $(25.8410)$ & $(26.3359)$ & $(26.2781)$ \\
& $(27.5294)$ & 27.2458 & 26.4083 & 26.1343 & 26.5497 & 27.4858 & 28.3854 \\
0 & $(27.5294)$ & $(26.5061)$ & 26.2665 & 25.5652 & $(25.8410)$ & $(26.3359)$ & 26.7064 \\
& 28.8696 & $(27.2458)$ & $(26.4083)$ & $(26.1343)$ & $(26.5497)$ & $(27.4858)$ & $(28.3854)$ \\
\hline \hline
\end{tabular}

TABLE III. Table of the lowest two energy levels for each $S_{T}^{z}, q$ for $N=10, S=\frac{3}{2}$.

\begin{tabular}{|c|c|c|c|c|c|c|}
\hline$S_{T}^{z}$ & 0 & $\pi / 5$ & $2 \pi / 5$ & $3 \pi / 5$ & $4 \pi / 5$ & $\pi$ \\
\hline 15 & $0^{-}$ & - & - & - & - & - \\
\hline 14 & (0) & $\overline{0.5729}$ & $\overline{2.0729}$ & $\overline{3.9271}$ & $\overline{5.4271}$ & $6^{-}$ \\
\hline 13 & $\begin{array}{l}10.1513 \\
11.7605\end{array}$ & $\begin{array}{r}8.8419 \\
10.8402\end{array}$ & $\begin{array}{r}9.3005 \\
10.6489\end{array}$ & $\begin{array}{l}7.6668 \\
8.9199\end{array}$ & $\begin{array}{l}7.1007 \\
7.7528\end{array}$ & $\begin{array}{c}6 \\
(6)\end{array}$ \\
\hline 12 & $\begin{array}{l}12.3285 \\
12.3396\end{array}$ & $\begin{array}{l}12.6499 \\
13.7320\end{array}$ & $\begin{array}{l}13.8552 \\
15.5538\end{array}$ & $\begin{array}{l}14.5474 \\
15.5100\end{array}$ & $\begin{array}{l}14.7933 \\
16.0547\end{array}$ & $\begin{array}{l}15.1047 \\
17.1664\end{array}$ \\
\hline 11 & $\begin{array}{l}19.7763 \\
22.1932\end{array}$ & $\begin{array}{l}19.3031 \\
20.9581\end{array}$ & $\begin{array}{l}19.4575 \\
20.2529\end{array}$ & $\begin{array}{l}18.7356 \\
19.9078\end{array}$ & $\begin{array}{l}18.1413 \\
20.1177\end{array}$ & $\begin{array}{l}18.2463 \\
18.3213\end{array}$ \\
\hline 10 & $\begin{array}{l}24.3389 \\
24.3494\end{array}$ & $\begin{array}{l}22.7534 \\
23.9652\end{array}$ & $\begin{array}{l}23.3125 \\
24.2412\end{array}$ & $\begin{array}{l}23.9641 \\
24.7285\end{array}$ & $\begin{array}{l}23.5178 \\
25.4926\end{array}$ & $\begin{array}{l}24.1754 \\
26.8390\end{array}$ \\
\hline 9 & $\begin{array}{l}28.2456 \\
31.0990\end{array}$ & $\begin{array}{l}27.4930 \\
29.6531\end{array}$ & $\begin{array}{l}28.1064 \\
28.8246\end{array}$ & $\begin{array}{l}27.4685 \\
28.3961\end{array}$ & $\begin{array}{l}27.8691 \\
28.2328\end{array}$ & $\begin{array}{l}27.6655 \\
27.7076\end{array}$ \\
\hline 8 & $\begin{array}{c}(31.0990) \\
31.1753\end{array}$ & $\begin{array}{l}31.1970 \\
31.7401\end{array}$ & $\begin{array}{l}31.7788 \\
32.1870\end{array}$ & $\begin{array}{l}31.8798 \\
32.5493\end{array}$ & $\begin{array}{l}31.1739 \\
33.4390\end{array}$ & $\begin{array}{l}31.9441 \\
34.9666\end{array}$ \\
\hline 7 & $\begin{array}{l}35.2624 \\
38.4366\end{array}$ & $\begin{array}{l}34.4751 \\
36.8433\end{array}$ & $\begin{array}{l}35.2739 \\
35.9175\end{array}$ & $\begin{array}{l}34.6185 \\
35.6272\end{array}$ & $\begin{array}{l}34.6480 \\
35.3498\end{array}$ & $\begin{array}{c}34.6560 \\
(34.9666)\end{array}$ \\
\hline 6 & $\begin{array}{c}37.7206 \\
(38.4366)\end{array}$ & $\begin{array}{l}37.5996 \\
38.6471\end{array}$ & $\begin{array}{l}37.5838 \\
38.7253\end{array}$ & $\begin{array}{l}38.2673 \\
38.9106\end{array}$ & $\begin{array}{l}37.8512 \\
39.8563\end{array}$ & $\begin{array}{l}38.2008 \\
41.5046\end{array}$ \\
\hline 5 & $\begin{array}{l}40.7810 \\
44.1653\end{array}$ & $\begin{array}{l}39.9270 \\
42.4693\end{array}$ & $\begin{array}{l}40.8900 \\
41.5013\end{array}$ & $\begin{array}{l}40.3322 \\
41.4230\end{array}$ & $\begin{array}{l}40.2260 \\
41.5681\end{array}$ & $\begin{array}{c}40.2921 \\
(41.5046)\end{array}$ \\
\hline 4 & $\begin{array}{c}42.5945 \\
(44.1653)\end{array}$ & $\begin{array}{c}(42.4693) \\
44.0649\end{array}$ & $\begin{array}{l}42.7758 \\
43.6709\end{array}$ & $\begin{array}{l}43.1428 \\
43.6759\end{array}$ & $\begin{array}{l}42.6520 \\
44.6771\end{array}$ & $\begin{array}{l}42.9291 \\
46.4108\end{array}$ \\
\hline 3 & $\begin{array}{l}44.7102 \\
48.2312\end{array}$ & $\begin{array}{c}(44.0649) \\
46.4739\end{array}$ & $\begin{array}{l}45.0377 \\
45.4387\end{array}$ & $\begin{array}{l}44.7418 \\
45.4356\end{array}$ & $\begin{array}{c}(44.6771) \\
46.1046\end{array}$ & $\begin{array}{c}44.5852 \\
(46.4108)\end{array}$ \\
\hline 2 & $\begin{array}{c}46.2499 \\
(48.2312)\end{array}$ & $\begin{array}{c}(46.4739) \\
47.6539\end{array}$ & $\begin{array}{l}46.3758 \\
46.7935\end{array}$ & $\begin{array}{l}46.4167 \\
46.7290\end{array}$ & $\begin{array}{c}(46.1046) \\
47.8513\end{array}$ & $\begin{array}{c}(46.4108) \\
49.6147\end{array}$ \\
\hline 1 & $\begin{array}{c}(48.2312) \\
50.5498\end{array}$ & $\begin{array}{c}(47.6539) \\
48.7697\end{array}$ & $\begin{array}{c}(46.7935) \\
47.4966\end{array}$ & $\begin{array}{c}(46.7290) \\
47.4709\end{array}$ & $\begin{array}{c}(47.8513) \\
48.7286\end{array}$ & $\begin{array}{c}47.1872 \\
(49.6147)\end{array}$ \\
\hline 0 & $\begin{array}{l}(48.2312) \\
(50.5498)\end{array}$ & $\begin{array}{l}(47.6539) \\
(48.7697)\end{array}$ & $\begin{array}{l}(46.7935) \\
(47.4966)\end{array}$ & $\begin{array}{c}47.0226 \\
(47.4709)\end{array}$ & $\begin{array}{l}(47.8513) \\
(48.7286)\end{array}$ & $\begin{array}{c}(49.6147) \\
51.0186\end{array}$ \\
\hline
\end{tabular}

TABLE IV. Table of the lowest two energy levels for each $S_{T}^{z}, q$ for $N=8, S=2$.

\begin{tabular}{|c|c|c|c|c|c|}
\hline$S_{T}^{z} q$ & 0 & $\pi / 4$ & $\pi / 2$ & $3 \pi / 4$ & $\pi$ \\
\hline 16 & $0^{-}$ & - & - & - & - \\
\hline
\end{tabular}


TABLE IV. (Continued).

\begin{tabular}{|c|c|c|c|c|c|}
\hline$q$ & 0 & $\pi / 4$ & $\pi / 2$ & $3 \pi / 4$ & $\pi$ \\
\hline 15 & (0) & $\overline{1.1716}$ & $4^{-}$ & $\overline{6.8284}$ & $8^{-}$ \\
\hline 14 & $\begin{array}{l}12.9275 \\
15.6155\end{array}$ & $\begin{array}{l}10.5091 \\
14.0281\end{array}$ & $\begin{array}{l}11.3901 \\
13.3471\end{array}$ & $\begin{array}{r}8.9738 \\
10.3155\end{array}$ & $\begin{array}{l}7 \\
8\end{array}$ \\
\hline 13 & $\begin{array}{l}16.3479 \\
16.5006\end{array}$ & $\begin{array}{l}17.0311 \\
19.4733\end{array}$ & $\begin{array}{l}18.4506 \\
19.8598\end{array}$ & $\begin{array}{l}18.7964 \\
20.9518\end{array}$ & $\begin{array}{l}19.3540 \\
22.7676\end{array}$ \\
\hline 12 & $\begin{array}{l}25.5872 \\
29.4459\end{array}$ & $\begin{array}{l}24.6353 \\
27.4390\end{array}$ & $\begin{array}{l}25.0088 \\
26.2329\end{array}$ & $\begin{array}{l}23.7512 \\
25.3132\end{array}$ & $\begin{array}{l}25.1946 \\
25.2125\end{array}$ \\
\hline 11 & $\begin{array}{l}30.3500 \\
30.4141\end{array}$ & $\begin{array}{l}30.5388 \\
31.1667\end{array}$ & $\begin{array}{l}31.0778 \\
32.2076\end{array}$ & $\begin{array}{l}30.3751 \\
33.4734\end{array}$ & $\begin{array}{l}31.4368 \\
35.6424\end{array}$ \\
\hline 10 & $\begin{array}{l}36.8387 \\
41.3508\end{array}$ & $\begin{array}{l}35.7941 \\
39.0467\end{array}$ & $\begin{array}{l}36.6481 \\
37.7606\end{array}$ & $\begin{array}{l}35.8936 \\
36.7504\end{array}$ & $\begin{array}{c}(35.6424) \\
35.7587\end{array}$ \\
\hline 9 & $\begin{array}{c}40.7826 \\
(41.3508)\end{array}$ & $\begin{array}{l}41.0057 \\
42.0731\end{array}$ & $\begin{array}{l}41.7216 \\
42.8810\end{array}$ & $\begin{array}{l}40.7620 \\
44.1475\end{array}$ & $\begin{array}{l}41.7970 \\
46.5668\end{array}$ \\
\hline 8 & $\begin{array}{l}46.3046 \\
51.2860\end{array}$ & $\begin{array}{l}45.3045 \\
48.7681\end{array}$ & $\begin{array}{l}46.3100 \\
47.5374\end{array}$ & $\begin{array}{l}45.5795 \\
47.0264\end{array}$ & $\begin{array}{c}45.3830 \\
(46.5668)\end{array}$ \\
\hline 7 & $\begin{array}{c}49.6265 \\
(51.2860)\end{array}$ & $\begin{array}{l}49.6701 \\
51.5067\end{array}$ & $\begin{array}{l}50.4141 \\
51.7109\end{array}$ & $\begin{array}{l}49.4075 \\
52.9029\end{array}$ & $\begin{array}{l}50.3410 \\
55.5043\end{array}$ \\
\hline 6 & $\begin{array}{l}53.8983 \\
59.2168\end{array}$ & $\begin{array}{l}53.0312 \\
56.5454\end{array}$ & $\begin{array}{l}54.0367 \\
55.3887\end{array}$ & $\begin{array}{l}53.2668 \\
55.4787\end{array}$ & $\begin{array}{c}53.4149 \\
(55.5043)\end{array}$ \\
\hline 5 & $\begin{array}{c}56.6759 \\
(59.2168)\end{array}$ & $\begin{array}{c}(56.5454) \\
58.9225\end{array}$ & $\begin{array}{l}57.1897 \\
58.5526\end{array}$ & $\begin{array}{l}56.1616 \\
59.6884\end{array}$ & $\begin{array}{l}56.9757 \\
62.4181\end{array}$ \\
\hline 4 & $\begin{array}{l}59.5699 \\
65.1019\end{array}$ & $\begin{array}{c}(58.9225) \\
62.3251\end{array}$ & $\begin{array}{l}59.8885 \\
61.1836\end{array}$ & $\begin{array}{c}(59.6884) \\
61.8182\end{array}$ & $\begin{array}{c}59.4210 \\
(62.4181)\end{array}$ \\
\hline 3 & $\begin{array}{c}61.6842 \\
(65.1019)\end{array}$ & $\begin{array}{c}(62.3251) \\
64.1480\end{array}$ & $\begin{array}{l}62.1261 \\
63.2680\end{array}$ & $\begin{array}{c}(61.8182) \\
64.4485\end{array}$ & $\begin{array}{c}(62.4181) \\
67.2608\end{array}$ \\
\hline 2 & $\begin{array}{c}(65.1019) \\
68.8878\end{array}$ & $\begin{array}{c}(64.1480) \\
66.0500\end{array}$ & $\begin{array}{l}63.8581 \\
64.8002\end{array}$ & $\begin{array}{c}(64.4485) \\
65.9032\end{array}$ & $\begin{array}{c}63.4384 \\
(67.2608)\end{array}$ \\
\hline 1 & $\begin{array}{l}(65.1019) \\
(68.8878)\end{array}$ & $\begin{array}{c}(66.0500) \\
67.0747\end{array}$ & $\begin{array}{c}(64.8002) \\
65.7905\end{array}$ & $\begin{array}{c}(65.9032) \\
67.1239\end{array}$ & $\begin{array}{c}(67.2608) \\
69.9763\end{array}$ \\
\hline 0 & $\begin{array}{c}(68.8878) \\
70.5219\end{array}$ & $\begin{array}{l}(66.0500) \\
(67.0747)\end{array}$ & $\begin{array}{l}(64.8002) \\
(65.7905)\end{array}$ & $\begin{array}{l}(65.9032) \\
(67.1239)\end{array}$ & $\begin{array}{l}(67.2608) \\
(69.9763)\end{array}$ \\
\hline
\end{tabular}

TABLE V. Table of the lowest two energy levels for each $S_{T}^{z}, q$ for $N=6, S=\frac{5}{2}$

\begin{tabular}{ccccc}
\hline \hline$S_{T}^{2} q$ & 0 & $\pi / 3$ & $2 \pi / 3$ & $\pi$ \\
\hline 15 & 0 & - & - & - \\
& - & - & - & - \\
14 & $(0)$ & - & 7.5 & 10 \\
& 14.4205 & 10 & 12.1081 & 9 \\
13 & 19.3951 & 16.5 & 14.5756 & 10 \\
& 21.1520 & 21.0541 & 20.9157 & 22.3150 \\
12 & 21.2345 & 22.7075 & 24.9915 & 28.1558 \\
& 29.9195 & 27.9326 & 29.2498 & 28.3375 \\
11 & 36.2715 & 32.8819 & 30.5762 & 28.5600 \\
& 35.7521 & 36.6231 & 35.1260 & 36.9196 \\
10 & $(36.2715)$ & 38.0180 & 40.1580 & 43.7346 \\
\hline
\end{tabular}


TABLE V. (Continued).

\begin{tabular}{lcccc}
\hline \hline$q$ & 0 & $\pi / 3$ & $2 \pi / 3$ & $\pi$ \\
\hline 9 & & & & 42.3989 \\
& 43.3569 & 41.8287 & 43.2463 & $(43.7346)$ \\
8 & 50.5395 & 46.8034 & 44.9666 & 49.1831 \\
& 48.5172 & 49.1845 & 48.0439 & 56.6813 \\
7 & $(50.5395)$ & 51.3118 & 52.8094 & 54.0078 \\
& 54.3974 & 53.6296 & 54.4734 & $(56.6813)$ \\
6 & 62.1555 & 58.1676 & 57.0027 & 58.9950 \\
& 58.8426 & 59.1205 & 58.5602 & 66.9575 \\
5 & $(62.1555)$ & 62.0105 & 62.8710 & 62.9754 \\
& 62.9827 & 62.7995 & 63.1284 & $(66.9575)$ \\
4 & 71.0830 & 66.9132 & 66.3165 & $(66.9575)$ \\
& 66.3826 & $(66.9132)$ & 66.3303 & 74.5277 \\
3 & $(71.0830)$ & 69.9066 & 70.2888 & 69.0673 \\
& $(71.0830)$ & $(69.9066)$ & $(70.2888)$ & $(74.5277)$ \\
2 & 77.2878 & 72.9932 & 72.7739 & $(74.5277)$ \\
& $(71.0830)$ & $(72.9932)$ & $(72.7739)$ & 79.3603 \\
1 & $(77.2878)$ & 74.9185 & 75.0237 & $(74.5277)$ \\
& $(77.2878)$ & $(74.9185)$ & $(75.0237)$ & $(79.3603)$ \\
0 & 80.7430 & 76.3782 & 76.3442 & $(79.3603)$ \\
\hline \hline & $(77.2878)$ & $(74.9184)$ & $(75.0237)$ & $(76.3442)$ \\
\hline
\end{tabular}

\section{APPENDIX B}

Tables VI-IX of correlation functions $F_{R}(N)=\left[\left\langle S_{i}^{z} S_{i+R}^{z}\right\rangle-\left(S_{T}^{z} / N\right)^{2}\right] / S^{2}$ for various $N, S, S_{T}^{z}$, and $R$.

TABLE VI. Values of $F_{R}(14)=\left(1 / S^{2}\right)\left[\left\langle S_{i}^{z} S_{i+R}^{z}\right\rangle-\left(S_{T}^{z} / N\right)^{2}\right]$ for $N=14, S=\frac{1}{2}$.

\begin{tabular}{|c|c|c|c|c|c|c|c|c|}
\hline$S_{T}^{2}{ }^{q}$ & 0 & 1 & 2 & 3 & 4 & 5 & 6 & 7 \\
\hline 0 & 1 & -0.5964 & 0.2484 & -0.2152 & 0.1544 & -0.1508 & 0.1280 & -0.1364 \\
\hline 1 & 0.9796 & -0.5388 & 0.1440 & -0.0780 & -0.0144 & 0.0376 & -0.0752 & 0.0700 \\
\hline 2 & 0.9184 & -0.4252 & -0.0136 & 0.0508 & -0.0752 & 0.0100 & 0.0216 & -0.0548 \\
\hline 3 & 0.8163 & -0.2957 & -0.1085 & 0.0315 & 0.0091 & -0.0437 & -0.0133 & 0.0239 \\
\hline 4 & 0.6735 & -0.1753 & -0.1161 & -0.0381 & 0.0107 & 0.0115 & -0.0145 & -0.0293 \\
\hline 5 & 0.4898 & -0.0802 & -0.0706 & -0.0534 & -0.0322 & -0.0126 & 0.0014 & 0.0062 \\
\hline 6 & 0.2653 & -0.0203 & -0.0203 & -0.0203 & -0.0203 & -0.0203 & -0.0203 & -0.0203 \\
\hline 7 & 0 & 0 & 0 & 0 & 0 & 0 & 0 & 0 \\
\hline
\end{tabular}

TABLE VII. Values of $F_{R}(12)=\left(1 / S^{2}\right)\left[\left\langle S_{i}^{z} S_{i+R}^{z}\right\rangle-\left(S_{T}^{z} / N\right)^{2}\right]$ for $N=12, S=1$.

\begin{tabular}{cccccrrr}
\hline \hline$R$ & 0 & 1 & 2 & 3 & 4 & 5 & \multicolumn{1}{c}{6} \\
$S_{T}^{z}$ & & & & & & \\
\hline 0 & 0.6667 & -0.4686 & 0.2592 & -0.2108 & 0.1681 & -0.1540 & 0.1454 \\
1 & 0.6082 & -0.3722 & 0.1494 & -0.1025 & 0.0524 & -0.0457 & 0.0293 \\
2 & 0.5648 & -0.3137 & 0.0856 & -0.0511 & 0.0059 & -0.0055 & -0.0076 \\
3 & 0.5180 & -0.2614 & 0.0379 & -0.0217 & -0.0075 & -0.0022 & -0.0083 \\
4 & 0.4680 & -0.2148 & 0.0060 & -0.0082 & -0.0100 & -0.0044 & -0.0051 \\
5 & 0.4144 & -0.1732 & -0.0124 & -0.0052 & -0.0087 & -0.0052 & -0.0049 \\
6 & 0.3579 & -0.1368 & -0.0214 & -0.0053 & -0.0077 & -0.0053 & -0.0049 \\
\hline
\end{tabular}


TABLE VII. (Continued).

\begin{tabular}{rccccccr}
\hline \hline$R$ & 0 & 1 & 2 & 3 & 4 & 5 & 6 \\
$S_{T}^{2}$ & & & & & & \\
\hline 7 & 0.3007 & -0.1059 & -0.0251 & -0.0052 & -0.0070 & -0.0050 & -0.0047 \\
8 & 0.2460 & -0.0784 & -0.0276 & -0.0044 & -0.0054 & -0.0055 & -0.0030 \\
9 & 0.1942 & -0.0516 & -0.0286 & -0.0087 & -0.0013 & -0.0038 & -0.0063 \\
10 & 0.1399 & -0.0258 & -0.0208 & -0.0139 & -0.0070 & -0.0020 & -0.0002 \\
11 & 0.0764 & -0.0070 & -0.0070 & -0.0070 & -0.0070 & -0.0070 & -0.0070 \\
12 & 0 & 0 & 0 & 0 & 0 & 0 \\
\hline \hline
\end{tabular}

TABLE VIII. Values of $F_{R}(10)=\left(1 / S^{2}\right)\left[\left\langle S_{i}^{z} S_{i+R}^{z}\right\rangle-\left(S_{T}^{z} / N\right)^{2}\right]$ for $N=10, S=\frac{3}{2}$.

\begin{tabular}{rcccccr}
\hline \hline$R$ & 0 & 1 & 2 & 3 & 4 & 5 \\
$S_{T}^{2}$ & & & & & & \\
\hline 0 & 0.5556 & -0.4225 & 0.2860 & -0.2572 & 0.2308 & -0.2299 \\
1 & 0.4706 & -0.3108 & 0.1530 & -0.1194 & 0.0850 & -0.0861 \\
2 & 0.4240 & -0.2539 & 0.0923 & -0.0641 & 0.0310 & -0.0346 \\
3 & 0.3872 & -0.2140 & 0.0556 & -0.0360 & 0.0078 & -0.0136 \\
4 & 0.3533 & -0.1816 & 0.0304 & -0.0207 & -0.0017 & -0.0063 \\
5 & 0.3205 & -0.1536 & 0.0130 & -0.0124 & -0.0052 & -0.0043 \\
6 & 0.2884 & -0.1291 & 0.0009 & -0.0079 & -0.0060 & -0.0041 \\
7 & 0.2568 & -0.1074 & -0.0072 & -0.0057 & -0.0060 & -0.0043 \\
8 & 0.2260 & -0.0880 & -0.0125 & -0.0048 & -0.0054 & -0.0045 \\
9 & 0.1955 & -0.0704 & -0.0155 & -0.0049 & -0.0048 & -0.0045 \\
10 & 0.1650 & -0.0544 & -0.0164 & -0.0053 & -0.0043 & -0.0042 \\
11 & 0.1344 & -0.0400 & -0.0156 & -0.0056 & -0.0040 & -0.0038 \\
12 & 0.1038 & -0.0272 & -0.0140 & -0.0057 & -0.0034 & -0.0034 \\
13 & 0.0732 & -0.0149 & -0.0109 & -0.0065 & -0.0032 & -0.0020 \\
14 & 0.04 & -0.0044 & -0.0044 & -0.0044 & -0.0044 & -0.0044 \\
15 & 0 & 0 & 0 & 0 & 0 & 0 \\
\hline \hline
\end{tabular}

TABLE IX. Values of $F_{R}(8)=\left(1 / S^{2}\right)\left[\left\langle S_{i}^{z} S_{i+R}^{z}\right\rangle-\left(S_{T}^{z} / N\right)^{2}\right]$ for $N=8, S=2$.

\begin{tabular}{rlrrrr}
\hline \hline$R$ & 0 & 1 & 2 & 3 & 4 \\
$S_{T}^{z}$ & & & & & \\
\hline 0 & 0.5 & -0.4013 & 0.3005 & -0.2838 & 0.2692 \\
1 & 0.3934 & -0.2729 & 0.1534 & -0.1344 & 0.1146 \\
2 & 0.3423 & -0.2140 & 0.0897 & -0.0727 & 0.0519 \\
3 & 0.3075 & -0.1771 & 0.0540 & -0.0414 & 0.0213 \\
4 & 0.2790 & -0.1497 & 0.0314 & -0.0242 & 0.0058 \\
5 & 0.2530 & -0.1272 & 0.0162 & -0.0147 & -0.0017 \\
6 & 0.2285 & -0.1080 & 0.0057 & -0.0095 & -0.0050 \\
7 & 0.2050 & -0.0910 & -0.0017 & -0.0068 & -0.0060 \\
8 & 0.1822 & -0.0759 & -0.0067 & -0.0055 & -0.0060 \\
9 & 0.1599 & -0.0623 & -0.0099 & -0.0050 & -0.0055 \\
10 & 0.1381 & -0.0500 & -0.0116 & -0.0050 & -0.0049 \\
11 & 0.1163 & -0.0389 & -0.0121 & -0.0050 & -0.0044 \\
12 & 0.0947 & -0.0287 & -0.0115 & -0.0051 & -0.0040 \\
13 & 0.0727 & -0.0195 & -0.0099 & -0.0050 & -0.0038 \\
14 & 0.0505 & -0.0113 & -0.0076 & -0.0046 & -0.0035 \\
15 & 0.0273 & -0.0039 & -0.0039 & -0.0039 & -0.0039 \\
16 & 0 & 0 & 0 & 0 & 0 \\
\hline \hline
\end{tabular}

* On leave from Department of Mathematics, University of Manchester, Institute of Science and Technology, P.O. Box 88, Manchester M60 1QD, United Kingdom.

$\dagger$ On leave from Department of Physics, University of Rhode Is- land, Kingston, RI 02881.

1J. C. Bonner, H. W. J. Blöte, H. Beck, and G. Müller, in Physics in One Dimension, edited by J. Bernasconi and T. Schneider (Springer, Berlin, 1981), p. 115; M. Steiner, J. Villain, and 
C. G. Windsor, Adv. Phys. 25, 87 (1976).

${ }^{2}$ F. D. M. Haldane, Phys. Lett. 93 A, 464 (1983); Phys. Rev. Lett. 50, 1153 (1983); Institut Laue-Langevin (Grenoble) Report No. SP81/95 (unpublished).

${ }^{3}$ See C. N. Yang and C. P. Yang, Phys. Rev. 151, 258 (1966), and related work.

${ }^{4}$ Symmetry properties of the Hamiltonian can be used to reduce substantially the dimensions of the matrices, but exponential growth with system size still results.

${ }^{5}$ C. Lanczös, J. Res. Natl. Bur. Stand. 45, 255 (1950); T. Sebe and J. Nachamkin, Ann. Phys. (N.Y.) 51, 100 (1969).

${ }^{6}$ R. Botet and R. Jullien, Phys. Rev. B 27, 613 (1983); M. Kolb, R. Botet, and R. Jullien, J. Phys. A 16, L673 (1983).

7J. C. Bonner and G. Müller, Phys. Rev. B 29, 5216 (1984).

${ }^{8}$ J. Sólyom and T. A. L. Ziman, Phys. Rev. B 30, 3980 (1984).

${ }^{9}$ S.-T. Chui and K. B. Ma, Phys. Rev. B 29, 1287 (1984).

10U. Glaus and T. Schneider, Phys. Rev. B 30, 215 (1984).

${ }^{11}$ (a) G. Müller, H. Beck, and J. C. Bonner, Phys. Rev. Lett. 43, 75 (1979); (b) G. Müller, H. Thomas, H. Beck, and J. C. Bonner, Phys. Rev. B 24, 1429 (1981).

${ }^{12}$ N. Ishimura and H. Shiba [Prog. Theor. Phys. 64, 479 (1980)] and T. Schneider and E. Stoll [Phys. Rev. B 25, 4721 (1982); 26, 3846 (1982)] also studied $S=\frac{1}{2}$ dynamics at low temperature using finite-chain calculations.

${ }^{13}$ G. Müller, H. Thomas, M. W. Puga, and H. Beck, J. Phys. C 14, 3399 (1981).

${ }^{14}$ However, the soft mode tracks from $q=\pi$ to $q=0$ in the case of $S^{z z}(q, \omega)$ and vice versa for $S^{x x}(q, \omega)$.

${ }^{15}$ Fowler and Puga were also aware of a soft mode (Kohn anomaly) whose location in the Brillouin zone was field dependent, but were unable to assess its importance for the dynamics. $M$. W. Puga, Phys. Rev. Lett. 42, 405 (1979); M. Fowler and M. W. Puga, Phys. Rev. B 19, 5906 (1979).

16J. des Cloizeaux and J. J. Pearson, Phys. Rev. 128, 2131 (1962).

17P. W. Anderson, Phys. Rev. 86, 694 (1952).

${ }^{18}$ See discussion and references in Ref. 11(b).

${ }^{19}$ J. Kurmann, H. Thomas, and G. Müller, Physica 112A, 235 (1982).

20J. H. Taylor and G. Müller, Phys. Rev. B 28, 1529 (1983).

${ }^{21}$ More progress in this direction has been made for the relatively easier static problems. Attempts to study Heisenberg AFM correlation functions and dynamics by the Bethe-ansatz, or, equivalently, the quantum inverse scattering, method have not, so far, been successful.

${ }^{22}$ See B. Sutherland, Phys. Rev. B 12, 3795 (1975).

${ }^{23}$ H. M. Babujian, Phys. Lett. 90A, 479 (1983); H. M. Babujian, Nucl. Phys. B 215, 317 (1983).

${ }^{24}$ L. A. Takhtajan, Phys. Lett. 87 A, 205 (1982).

${ }^{25}$ K. Sogo, Phys. Lett. 104 A, 51 (1984).

${ }^{26}$ H. Aghahosseini and J. B. Parkinson, J. Phys. C 14, 425 (1981).

${ }^{27}$ R. Botet, R. Jullien, and M. Kolb, Phys. Rev. B 28, 3914 (1983).

${ }^{28}$ H. J. Mikeska, Phys. Rev. B 12, 2794 (1975); H. C. Fogedby, J. Phys. C 11, 4767 (1978).

${ }^{29} \mathrm{Kolb}$, Botet, and Jullien (Ref. 6) claim to have information on spin systems of comparable size. However, for $S=\frac{1}{2}$, they present data only on systems up to $N=12$.
${ }^{30}$ H. W. J. Blöte, Physica 79B, 427 (1975).

${ }^{31}$ J. J. Smit, L. J. de Jongh, H. W. J. Blöte, and D. de Klerk, International Colloquium of the CNRS, Grenoble (1974), No. 242, p. 271 (unpublished).

32J. C. Bonner and M. E. Fisher, Phys. Rev. 135, A640 (1964).

${ }^{33}$ R. B. Griffiths, Phys. Rev. 133, A768 (1964).

34J. B. Parkinson, J. Phys. C (to be published).

35J. B. Parkinson and J. C. Bonner, in Proceedings of the Workshop on Elementary Excitations and Fluctuations in Magnetic Systems, edited by S. W. Lovesey, U. Balucani, F. Borsa, and V. Tognetti (Springer, Berlin, 1984), p. 55. A preliminary version of our major conclusions is presented in this reference.

${ }^{36}$ Note that this normalization is convenient, but not essential to our argument.

${ }^{37}$ The $\Delta(0,0)$ gap is an exception.

${ }^{38}$ In fact, this is true for all spin values.

${ }^{39}$ L. Hulthén, Ark. Mat. Astron. Fys. 26 A, 1 (1938).

${ }^{40}$ M. Takahashi, J. Phys. C 10, 1289 (1977).

${ }^{41}$ R. J. Baxter, J. Stat. Phys. 9, 145 (1973).

42J. C. Bonner, J. Appl. Phys. 49, 1299 (1978).

${ }^{43}$ W. Grieger, Phys. Rev. B 30, 344 (1984).

44J. Borysowicz, T. A. Kaplan, and P. Horsch, unpublished work.

${ }^{45}$ A. Luther and I. Peschel, Phys. Rev. B 12, 3908 (1975).

46J. E. Hirsch, R. L. Sugar, D. J. Scalapino, and R. Blankenbecler, Phys. Rev. B 26, 5033 (1982).

${ }^{47}$ V. J. Emery (private communication); D. J. Scalapino (private communication) (to T. Kaplan).

${ }^{48}$ P. Horsch and T. A. Kaplan, J. Phys. C 16, L1203 (1983).

${ }^{49}$ F. D. M. Haldane, Phys. Rev. Lett. 45, 1358 (1980).

${ }^{50}$ E. Pytte, Phys. Rev. B 10, 4637 (1974).

${ }^{51}$ N. Ishimura and H. Shiba, Prog. Theor. Phys. 57, 1862 (1977).

${ }^{52} \mathrm{H}$. Aghahosseini and J. B. Parkinson, J. Phys. C 13, 651 (1980).

${ }^{53}$ Y. Wang and H. B. Callen, J. Phys. Chem. Solids 25, 1459 (1964).

${ }^{54}$ Some preliminary results for $S=1$ Heisenberg ferromagnets in zero field are found in G. Müller and H. Beck, J. Phys. C 11,483 (1978).

${ }^{55}$ See Ref. 12.

56E. Barouch and B. M. McCoy, Phys. Rev. A 3, 786 (1971).

${ }^{57} \mathrm{~A}$ conjecture by Müller et al. [Ref. 11(b)] that the correlations should behave as $\left\langle S_{i}^{z} S_{i+R}^{z}\right\rangle \sim \cos \left(q_{P} R\right) / R^{2}$, where $q_{P}=\pi(1-2 M)$, is not consistent with the Haldane work, of Ref. 49, and is in moderate qualitative agreement with our extrapolations.

${ }^{58}$ Note that for finite $N, S(q)$ remains finite for $q \rightarrow \pi$, but a divergence is expected in the thermodynamic limit for $S=\frac{1}{2}$. The behavior of $S(\pi)$ for $S>\frac{1}{2}$ is intimately related to the validity of the Haldane conjecture [J. B. Parkinson, J. C. Bonner, G. Müller, M. P. Nightingale, and H. W. J. Blöte, J. Appl. Phys. 57, 3319 (1984)].

${ }^{59}$ Our Fig. 11 is based on a figure of I. U. Heilman, G. Shirane, Y. Endoh, R. J. Birgeneau, and S. L. Holt, Phys. Rev. B 18, 3530 (1978).

${ }^{60}$ C. S. Jain, K. Krishan, C. K. Majumdar, and V. Mubayi, Phys. Rev. B 12, 5235 (1975).

${ }^{61}$ G. Müller, Phys. Rev. B 26, 1311 (1982). 